\title{
Detecting and switching magnetization of Stoner nanograin in nonlocal spin valve
}

\author{
Hai-Zhou Lu (卢海舟) and Shun-Qing Shen (沈顺清) \\ Department of Physics and Centre of Theoretical and Computational Physics, The University of Hong Kong, Pokfulam Road, \\ Hong Kong, China
}

(Received 5 March 2009; published 2 September 2009)

\begin{abstract}
The magnetization detection and switching of an ultrasmall Stoner nanograin in a nonlocal spin valve (NLSV) device is studied theoretically. With the help of the rate equations, a unified description can be presented on the same footing for the NLSV signal that reads out the magnetization, and for the switching process. The setup can be viewed as that the grain is connected to two nonmagnetic leads via sequential tunneling. In one lead, the chemical potentials for spin-up and -down electrons are split due to the spin injection in the NLSV. This splitting (or the spin bias) is crucial to the NLSV signal and the critical condition to the magnetization switching. By using the standard spin diffusion equation and parameters from recent NLSV device, the magnitude of the spin bias is estimated and found large enough to drive the magnetization switching of the cobalt nanograin reported in earlier experiments. A microscopic interpretation of NLSV signal in the sequential tunneling regime is thereby raised, which show properties due to the ultrasmall size of the grain. The dynamics at the reversal point shows that there may be a spin-polarized current instead of the anticipated pure spin current flowing during the reversal due to the electron accumulation in the floating lead used for the readout of NLSV signal.
\end{abstract}

DOI: 10.1103/PhysRevB.80.094401

\section{INTRODUCTION}

Current-induced magnetization reversal had attracted considerable interest due to its fundamental significance in understanding interplay between magnetism and electricity as well as potential applications in magnetic memories. ${ }^{1-7}$ As the scale of the ferromagnetic nanograins goes down to only several nanometers, ${ }^{8-11}$ many theoretical ${ }^{12-21}$ and experimental works ${ }^{22-25}$ were inspired to address the current-induced magnetization reversal in these small structures. By far, most studied setups were multilayer or nanopillar structures with vertical geometries in which spins are always carried along the flowing of charge current. Usually, the critical current density as high as $10^{6}-10^{9} \mathrm{~A} / \mathrm{cm}^{2}$ is required to induce a reversal. ${ }^{26}$ Considering such high density of current flowing through each nanograin, when a huge amount of nanograins are integrated in large scale spurious effects such as Joule heat, current-induced magnetic field, and noise are not ignorable.

A possible solution is to use pure spin current in which the same amount of spin-up and -down currents flow along opposite directions, yielding no net electric current. By far, one of the most promising designs to realize considerable pure spin current is the nonlocal spin valve (NLSV) devices $^{27-34}$ with lateral geometry. ${ }^{35}$ Recently, a NLSV was reported to reversibly switch the magnetization of a ferromagnetic particle. ${ }^{36,37}$ As shown in Fig. 1(a), a typical NLSV includes a bigger fixed and a smaller free ferromagnets (denoted by the shadow areas) embedded on the left and right sides, usually referred as injector and detector, respectively. By driving a current $I_{c}$ through regions (2), (1), and (3), spins can be injected from injector (1) to produce a nonequilibrium spin accumulation in nonmagnetic region (4). This spin accumulation exhibits as a splitting of chemical potentials for spin-up and -down electrons (spin bias or spin voltage), as shown in Figs. 3(a) and 3(b). While the spin-polarized current flows only in the loop formed by regions (2), (1), and
PACS number(s): 85.75.-d, 73.23.-b, 72.25.Hg, 85.35.-p

(3), the nonequilibrium spin accumulation in region (4) diffuses to the right accompanied by a pure spin current flowing toward the detector. In this way, net charge current is prevented from flowing directly into the grain. In the response of $I_{c}$, the magnetization of the detector can be read out by measuring the voltage difference $\Delta V$ between (5) and (6), referred to as the NLSV voltage, which is usually estimated by the spin diffusion equation. ${ }^{38-42}$ By applying $I_{c}$ exceeding a critical value, the magnetization of the detector can be switched reversibly. ${ }^{36,37}$ This process is usually described by Landau-Lifshitz-Gilbert equation. ${ }^{43}$

By far, the detectors are films of size of $100-1000 \mathrm{~nm}$. Considering commercial charge devices already work well at these scales, the ultimate goal of utilizing pure spin degree of freedom is to replace the charge devices at only several nanometers. Besides, because the LLG equation preassume the magnitude and polarization of currents that flow through the grain as input parameters, the counteraction of the detector on the currents is not taken into account. As a result, important information could be missing, e.g., whether the pure spin current is still a pure spin current after flowing into the detector.

In this work, we study a NLSV device in which the usual film detector is replaced with one or multiple well-separated cobalt nanograins embedded in insulator, as those in Ref. 9. The nanograin is much smaller in size $\left(\sim 10^{3}\right.$ atoms $)$ than the films and at much lower temperatures $(\sim 20 \mathrm{mK})$. Such small nanograin can be viewed as a Stoner particle whose ferromagnetism comes from the exchange interactions between itinerant electrons inside it, and thereby can be manipulated by exchanging angular momenta with the electrons that tunnel through it. ${ }^{16,44}$ The grain is modeled as coupled to two nonmagnetic leads via quantum tunneling. In one lead, the chemical potentials for the spin-up and -down electrons are split due to the spin injection in the NLSV. The small size of the nanograin allows us to model the NLSV signal and the magnetization switching within one set of rate equations. Be- 

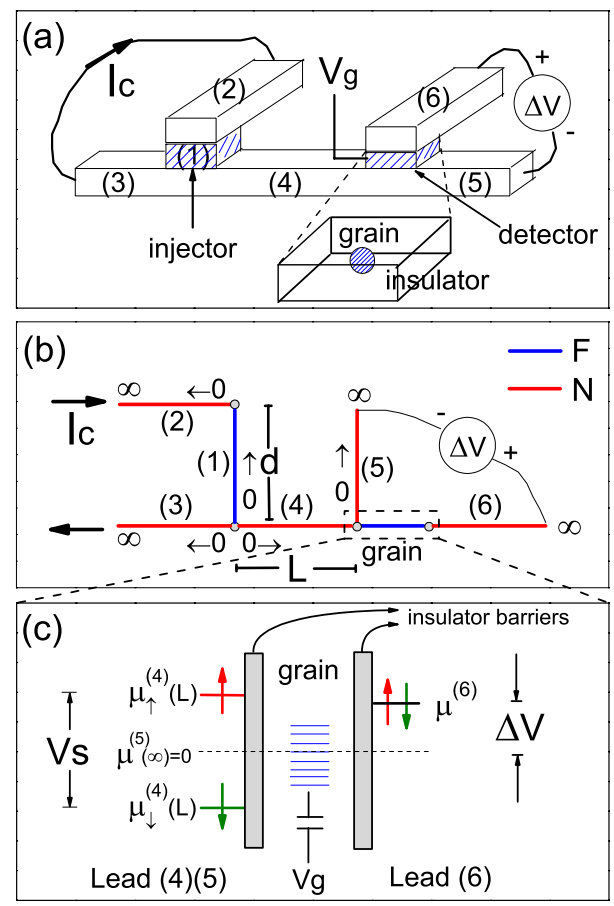

FIG. 1. (Color online) (a) The schematic of our nonlocal spin valve (NLSV) device. It can be viewed as a combination of the setups employed in Ref. 9 and 37. (b) The device is separated into seven regions. (1) is a fixed ferromagnetic injector of thickness $d$. The ferromagnetic grain on the right is to be manipulated and detected. The distance between (1) and the grain is $L$. " $0 \rightarrow$ " in each region defines the local origin and positive direction of coordinate. (c) The grain and its nearby regions is modeled as a Stoner particle coupled to two nonmagnetic lead via tunneling through two barriers. Driven by the spin-polarized current $I_{c}$, spins injected from (1) accumulate and induce a splitting $\left(V_{s}\right)$ of the spin-up and -down chemical potentials $\left[\mu_{\uparrow / l}^{(4)}(L)\right]$ at the (4)/(5)/grain interface. $V_{g}$ is the gate voltage applied to the insulator [see Fig. 1(a)] surrounding the grain, much like the technique in Ref. 9. In this way, it can be used to tune the energy levels inside the grain with respect to chemical potentials of (4)-(6) capacitively, while without inducing direct current between the gate and the grain. $\Delta V$ measures the voltage between (5) and (6) and is defined as the NLSV voltage. $\mu^{(5)}(\infty)$ is the chemical potential at the voltmeter side of regions (5) and is set as the energy zero point throughout the paper.

sides, the previous knowledge of the Co nanograin from experiments $^{8-11}$ and theories ${ }^{45,46}$ allows us to perform a realistic evaluation.

This work focuses on two aspects: (i) the possibility of the detection and switching is evaluated using the parameters extracted from the previous NLSV (Refs. 36 and 37) and Co grain $^{8-11}$ experiments. (ii) Such small grain is subjected to strong Coulomb and magnetic blockades, ${ }^{16}$ how these blockades determine the critical conditions for the reversal, e.g., the critical driving current $I_{c}$, the gate voltage $V_{g}$, and the spin bias $V_{s}$.

We find that: (i) the numerical evaluations using realistic parameters from the recent NLSV (Refs. 36 and 37) and the cobalt grain experiments $^{8-11}$ show that it is possible to employ the NLSV device to detect and switch the magnetization of a ferromagnetic nanograin under the present experimental conditions. (ii) Under $I_{c}$, the NLSV signal can also be detected in the sequential tunneling regime to read out the magnetization of the grain and interpreted from a microscopic view. Interestingly, if the majority band of the grain is favored to participate in the electron transport, the sign of the NLSV signal turns out to be just opposite to that if the minority band is preferred to conduct electrons. In the presence of an angle $\theta$ between the easy axis of the grain and the spin-quantization direction of the lead, the NLSV signal is proportional to $\cos \theta$ and vanishes when $\theta=\pi / 2$. (iii) Under $I_{c}$ exceeding a critical value, the magnetization of the grain can be switched reversibly. The critical current $I_{c}$ required for the magnetization switching is determined by the gate voltage $V_{g}$ and the spin bias $V_{s}$ at which both the Coulomb and magnetic blockades in the grain are lifted. By choosing suitable gate voltage, the critical $V_{s}$ needed for switching can be minimized to $\sim 2 K_{N}$, where $K_{N}$ is the volume-independent anisotropy of the grain. Besides, the transient current during the magnetization reversal may be a spin-polarized current instead of the anticipated pure spin current due to the electron accumulation or drainage in the floating lead used for the NLSV measurement. A possible solution is to remove the floating lead.

The paper is organized as follows. In Sec. II, the model and theoretical formalisms are introduced. In Sec. III, the microscopic NLSV signal in the sequential tunneling regime is described in detail. In Sec. IV, the magnetization switching under large injection current $I_{c}$ is presented. Finally, a summary is given in Sec. V.

\section{MODEL AND THEORETICAL FORMALISMS}

\section{A. General survey of our setup}

The device we study is shown in Fig. 1(a), which can be divided into seven regions, "(1)-(6)" and "grain," as shown in Fig. 1(b). The different regions of the device are separated into three parts and modeled by different formalisms, depending on their sizes and positions:

(i) the first part consists of regions (1)-(5). Their sizes are comparable to their spin-diffusion lengths; thus, the spin transport in these regions are governed by the spin diffusion equations. ${ }^{38-42}$ The magnetization of injector (1) is assumed to be fixed.

(ii) The second part is the smaller (approximately several $\mathrm{nm}$ ) grain, which is described as a Stoner particle, ${ }^{45,46}$ coupled via sequential tunneling to two nonmagnetic leads. For convenience, we call them lead $(4,5)$ and lead (6), respectively. Lead $(4,5)$ and lead (6) are defined as where regions (4) and (5) and region (6) connect the grain, respectively. We assume that there is no direct tunneling between lead $(4,5)$ and lead (6); i.e., the only possible connection between them is via the grain.

(iii) The third part is floating region (6). Because of the voltmeter, it is an open circuit between regions (4) and (5) and region (6); i.e., at steady state, there is no current flowing from (4) and (5) across the grain to (6) due to a voltage difference between (6) and (5). Throughout the paper, we define the chemical potential $\mu^{(5)}(\infty)$ at the voltmeter side of region (5) as the energy zero point. The chemical potential of 


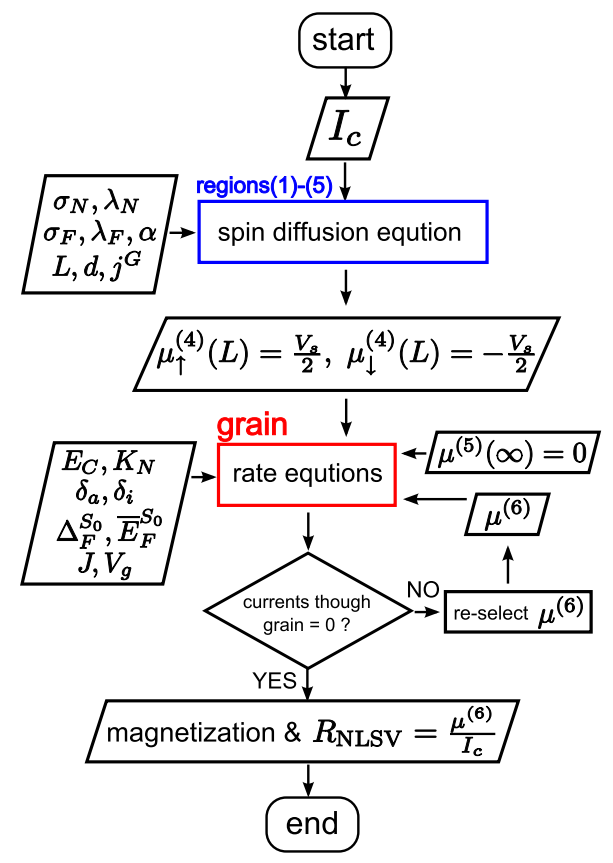

FIG. 2. (Color online) The calculation steps of the magnetization of the grain and the nonlocal spin valve signal $R_{\mathrm{NLSV}}$ as functions of $I_{c}$, the driving current. Rectangles represent calculation processes. Parallelograms represent inputs or outputs of the calculations. Regions (1)-(5) in Fig. 1 are described by the spin diffusion equation given by Eq. (2). The grain and its nearby regions are described by the rate equations given by Eq. (12). The diamond represents the self-consistent calculation of $\mu^{(6)}$, the chemical potential of region (6).

region (6) is denoted as $\mu^{(6)}$. The difference between $\mu^{(6)}$ and $\mu^{(5)}(\infty)$ is denoted as $\Delta V \equiv \mu^{(6)}-\mu^{(5)}(\infty)$, where $\Delta V$ is the NLSV voltage. $\Delta V$ will be determined through selfconsistent calculation.

In a word, our model can be viewed as a combination of the setup used in two kinds of experiments, i.e., the NLSV (Ref. 37) and the transport through ferromagnetic nanograins. ${ }^{8,9}$ Besides:

(i) different from usual ferromagnetic nanograins setups, ${ }^{8,9}$ the chemical potentials for spin-up and -down electrons $\mu_{\uparrow / l}^{(4)}(L)$ are split at the $(4) /(5) /$ grain interface, as shown in Fig. 1(c). The splitting is called the spin bias, and denoted as $V_{s}{ }^{20,21,47-50}$ This spin bias is induced by the spins injected from region (1) to region (4). Later in Secs. III and IV A, we will see that $V_{s}$ is crucial to the detection of NLSV signal and the magnetization reversal; thus, we will first evaluate its magnitude in the following subsection.

(ii) Different for usual NLSVs, ${ }^{36,37}$ The ultrasmall size of the grain allows the NLSV signal, the magnetization reversal dynamics, and the interaction between the currents and the grain to be described within one set of rate equations [Eq. (12)].

For a better understanding of our setup, the calculation steps of the magnetization and the NLSV signal as functions of $I_{c}$ is presented in Fig. 2. This section is organized as follows. In Sec. II B, $V_{s}$ will be estimated with realistic experimental parameters. In Sec. II C, the description of the grain will be introduced. In Sec. II D, the transport between the grain and its leads will be described by the rate equations. The details of theoretical descriptions can be found in Appendixes $\mathrm{A}$ and $\mathrm{C}$ and will be specified in the following subsections.

\section{B. Spin diffusion transport in regions (1)-(5) and estimate of spin bias $V_{s}$}

In this section, we will estimate the magnitude of $V_{s}$, which is defined as the splitting of spin-up and -down chemical potentials at the $(4) /(5) /$ grain interface,

$$
V_{s} \equiv \mu_{\uparrow}^{(4)}(L)-\mu_{\downarrow}^{(4)}(L) \equiv \mu_{\uparrow}^{(5)}(0)-\mu_{\downarrow}^{(5)}(0) .
$$

Following the previous literatures, the spin and charge transports in regions (1)-(5) are described by the standard diffusion equation, ${ }^{38-42}$

$$
\begin{gathered}
\nabla^{2}\left(\sigma_{\uparrow} \mu_{\uparrow}+\sigma_{\downarrow} \mu_{\downarrow}\right)=0, \\
\nabla^{2}\left(\mu_{\uparrow}-\mu_{\downarrow}\right)=\frac{1}{\lambda^{2}}\left(\mu_{\uparrow}-\mu_{\downarrow}\right),
\end{gathered}
$$

where $\lambda$ is a phenomenological spin-diffusion length that can be measured experimentally. ${ }^{51}$ Usually, the spin-diffusion length in normal metal is much longer than that in ferromagnetic metal, i.e., $\lambda_{N} \gg \lambda_{F}$. In this work, we use $\lambda_{N}$ $=1000 \mathrm{~nm}$ and $\lambda_{F}=5 \mathrm{~nm}^{37} \sigma_{\sigma}$ is the conductivity for $\sigma$ electrons. For ferromagnetic metal, $\sigma_{\uparrow}=\sigma_{F}(1+\alpha) / 2$ and $\sigma_{\downarrow}$ $=\sigma_{F}(1-\alpha) / 2$, where $\sigma_{F}$ is the total conductivity of the ferromagnetic metal, $\alpha$ is the bulk polarization defined as $\alpha$ $\equiv\left(\sigma_{\uparrow}-\sigma_{\downarrow}\right) /\left(\sigma_{\uparrow}+\sigma_{\downarrow}\right)$. For normal metal, $\sigma_{\uparrow}=\sigma_{\downarrow}=\sigma_{N} / 2$. In this work, we use $\sigma_{N}=8.8 \times 10^{7} \Omega^{-1} \mathrm{~m}^{-1}, \quad \sigma_{F}=9.8$ $\times 10^{6} \Omega^{-1} \mathrm{~m}^{-1}$, and $\alpha=0.2 .^{36}$

We calculate the chemical potentials in regions (1)-(5) of Fig. 1(b), where regions (2), (3), and (5) can be regarded as semi-infinite. By assuming that the same cross-section area $A_{\square}$ perpendicular to the current in each region, the solutions to Eq. (2) can be simplified to be one dimensional. Following Jedema et al. ${ }^{41}$ the general solutions to the 5 regions as functions of position $x$ are given by

$$
\begin{gathered}
\mu_{\uparrow / \downarrow}^{(1)}=A+\frac{e j_{c}}{\sigma_{F}} x \pm \frac{B}{\sigma_{F}(1 \pm \alpha)} e^{-x / \lambda_{F}} \pm \frac{C}{\sigma_{F}(1 \pm \alpha)} e^{x / \lambda_{F}}, \\
\mu_{\uparrow \uparrow \downarrow}^{(2)}=D+\frac{e j_{c}}{\sigma_{N}} x \pm \frac{E}{\sigma_{N}} e^{-x / \lambda_{N}}, \\
\mu_{\uparrow \uparrow \downarrow}^{(3)}=-\frac{e j_{c}}{\sigma_{N}} x \pm \frac{F}{\sigma_{N}} e^{-x / \lambda_{N}}, \\
\mu_{\uparrow \uparrow \downarrow}^{(4)}= \pm \frac{G}{\sigma_{N}} e^{-x / \lambda_{\mathrm{N}}} \pm \frac{H}{\sigma_{N}} e^{x / \lambda_{N},} \\
\mu_{\uparrow / \downarrow}^{(5)}= \pm \frac{K}{\sigma_{N}} e^{-x / \lambda_{\mathrm{N}}},
\end{gathered}
$$


where the origin and positive direction in each region are locally defined for a concise form of the general solutions and indicated by " $0 \rightarrow$ " in Fig. $1(\mathrm{~b}) . j_{c}=I_{c} / A_{\square}$, where $I_{c}$ is the spin-polarized total current flowing through regions (2), (1), and (3). $e$ is the electron charge. The nine coefficients $A$, $B, C, D, E, F, G, H$, and $K$ will be determined by boundary conditions.

The boundary conditions are given as follows:

(i) the current polarization usually loses when flowing through an interface from the ferromagnetic to the normal side due to, e.g., the spin-dependent scattering. We take this loss into account by phenomenologically introducing an efficiency parameter $\gamma \in[0,1]$,

$$
\gamma \frac{j_{F \uparrow}-j_{F \downarrow}}{j_{F \uparrow}+j_{F \downarrow}}=\frac{j_{N \uparrow}-j_{N \downarrow}}{j_{N \uparrow}+j_{N \downarrow}}
$$

where $j_{\sigma}=-\left(\sigma_{\sigma} / e\right) \partial_{x} \mu_{\sigma}$ is the current density for the spin- $\sigma$ electrons. By combining Eq. (4) and the conservation of the total current

$$
j_{F \uparrow}+j_{F \downarrow}=j_{N \uparrow}+j_{N \downarrow},
$$

the boundary conditions at the ferromagnetic/normal interface are then given by

$$
\begin{gathered}
j_{F \uparrow}=\frac{\gamma+1}{2 \gamma} j_{N \uparrow}+\frac{\gamma-1}{2 \gamma} j_{N \downarrow}, \\
j_{F \downarrow}=\frac{\gamma+1}{2 \gamma} j_{N \downarrow}+\frac{\gamma-1}{2 \gamma} j_{N \uparrow} .
\end{gathered}
$$

(ii) The chemical potentials of each spin components are continuous at each interface. For simplicity, we do not explicitly include the spin-dependent chemical-potential drops caused by the interface resistance. Its destructive effects, in particular on the reduction in the spin bias, will be approximately accounted by considering a relatively small injection efficiency $\gamma$.

(iii) At the (4)/(5)/grain interface, the spin-up and -down currents flowing into the grain are $I^{G}$ and $-I^{G}$, respectively, i.e., (how this boundary condition is derived can be found in Appendix A)

$$
-A_{\square} \frac{\sigma_{N}}{2} \partial_{x} \mu_{\uparrow / \downarrow}^{(4)}(L)= \pm e I^{G}-A_{\square} \frac{\sigma_{N}}{2} \partial_{x} \mu_{\uparrow / \downarrow}^{(5)}(0),
$$

where we assume a pure spin current flowing from (4) and (5) into the grain, which however may not be true as we will see in Sec. IV B. However, this deviation is neglected because $I^{G}$ is too small to affect $V_{s}$, as we will see in Sec. IV C. Actually, because $I^{G}$ is ignorably small, we simply neglect it in our numerical calculations, though we include it in equations explicitly.

With these boundary conditions, the coefficients from $A$ through $K$ in Eq. (3) are readily found. Figures 3(a) and 3(b) show the spin-resolved chemical potentials $\mu_{\uparrow}$ and $\mu_{\uparrow}$ in regions (1)-(5). For a clear demonstration of the splitting between spin-up and -down chemical potentials, we choose $\alpha$ $=0.9$ only in these two figures. For the realistic evaluations
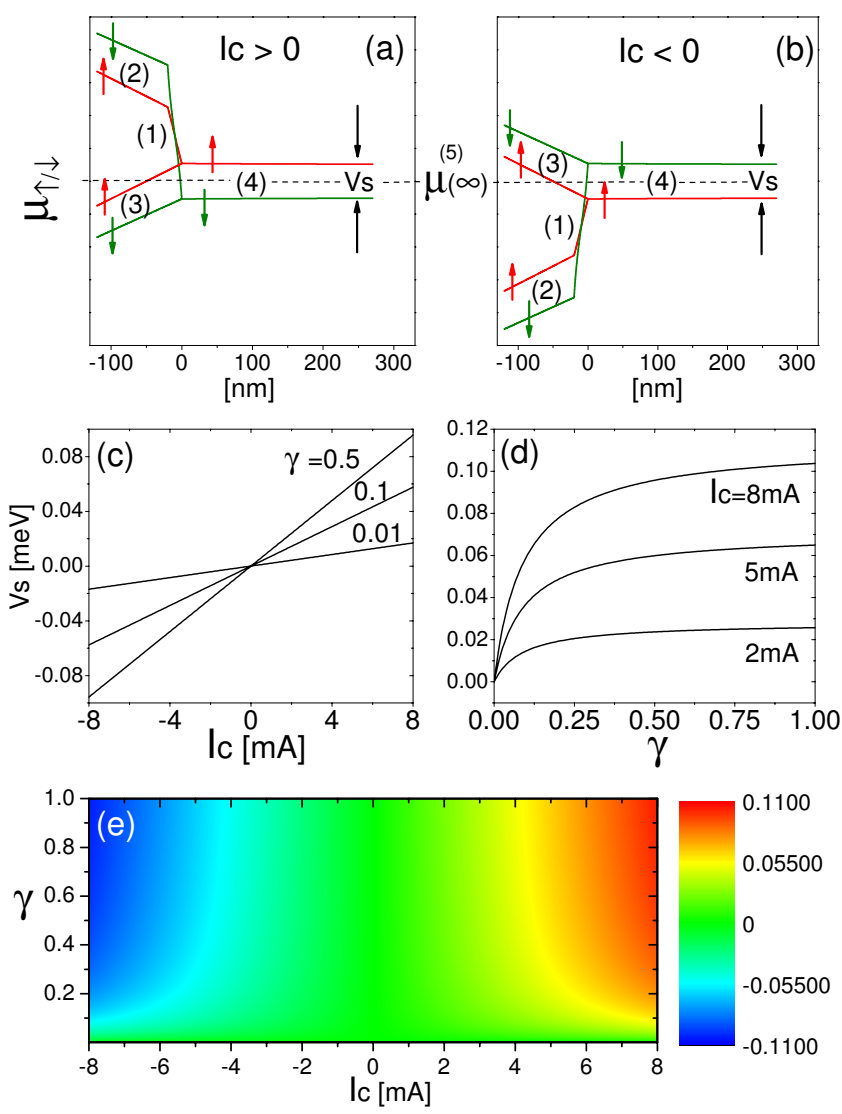

FIG. 3. (Color online) (a) and (b) The chemical potentials $\mu_{\uparrow}$ (marked by $\uparrow$ ) and $\mu_{\downarrow}$ (marked by $\downarrow$ ) in regions (1)-(4). They change with the direction of $I_{c}$, the spin-polarized current flowing through regions (1)-(3). $V_{s}$ is defined as $\mu_{\uparrow}-\mu_{\downarrow}$ at the (4)/(5)/grain interface. The horizontal dashed line indicates $\mu^{(5)}(\infty)$, the energy zero point throughout the paper. (c) $V_{s}$ vs $I_{c}$ for different values of spin injection efficiency $\gamma$. (d) $V_{s}$ vs $\gamma$ for different $I_{c}$. (e) $V_{s}$ (in unit of meV) as a function of $I_{c}$ and $\gamma$. The parameters for (c)-(e): $d$ $=20 \mathrm{~nm}, \quad L=270 \mathrm{~nm}, \quad A_{\square}=170 \times 65 \mathrm{~nm}^{2}, \quad \lambda_{F}=5 \mathrm{~nm}, \quad \lambda_{N}$ $=1000 \mathrm{~nm}, \quad \sigma_{F}=9.8 \times 10^{6} \Omega^{-1} \mathrm{~m}^{-1}, \quad \sigma_{N}=8.8 \times 10^{7} \Omega^{-1} \mathrm{~m}^{-1}, \quad \alpha$ $=0.2$, and $I^{G}=0$.

in the rest part of this work, we choose $\alpha=0.2$, as estimated by the experiments. ${ }^{36,37}$ All the conductivities, spin-diffusion lengths, and the bulk polarization used for the evaluation are extracted from the experiment data ${ }^{36,37}$ and given in Fig. 3 . Driven by $I_{c}$, the spins injected from injector (1) induce a splitting between $\mu_{\uparrow}$ and $\mu_{\downarrow}$ at interfaces (1)/(2) and (1)/(3)/ (4). Because injector (1) is sandwiched between two normal metals, $\mu_{\uparrow}$ and $\mu_{\downarrow}$ in (1) cross with each other at the center and split oppositely on the opposite sides. In each region of (1)-(3) because of the charge current $I_{c}, \mu_{\uparrow}$, and $\mu_{\downarrow}$ not only split but also demonstrate steep slopes with the same trend. Because of no net charge current, $\mu_{\uparrow}$ and $\mu_{\downarrow}$ in region (4) just diffuse to the right side in opposite gradients (not obvious here because $\left.L \ll \lambda_{N}\right)$.

By using Eq. (1), the analytic expression for $V_{s}$ is found out as

$$
V_{s} \equiv \mu_{\uparrow}^{(4)}(L)-\mu_{\downarrow}^{(4)}(L) \equiv \mu_{\uparrow}^{(5)}(0)-\mu_{\downarrow}^{(5)}(0),
$$




$$
\begin{aligned}
= & 2 e j_{c} \frac{\lambda_{F}}{\sigma_{F}} \frac{\alpha}{\left(1-\alpha^{2}\right)} \frac{\left(M_{1}-2\right)}{M_{2}} e^{-L / \lambda_{N}} \\
& -2 e \frac{I^{G}}{A_{\square}}\left[\frac{\lambda_{F}}{\sigma_{F}} \frac{2 e^{-2 L / \lambda_{N} M_{1}}}{\gamma\left(1-\alpha^{2}\right) M_{2}}+\frac{\lambda_{N}}{\sigma_{N}}\left(1-e^{\left.-2 L / \lambda_{N}\right)}\right],\right.
\end{aligned}
$$

where

$$
\begin{gathered}
M_{1}=2 \cosh \left(\frac{d}{\lambda_{F}}\right)+2 M_{0} \sinh \left(\frac{d}{\lambda_{F}}\right), \\
M_{2}=6 M_{0} \cosh \left(\frac{d}{\lambda_{F}}\right)+2\left(2 M_{0}^{2}+1\right) \sinh \left(\frac{d}{\lambda_{F}}\right), \\
M_{0}=\frac{\sigma_{N} \lambda_{F}}{\sigma_{F} \lambda_{N} \gamma\left(1-\alpha^{2}\right)} .
\end{gathered}
$$

Figures 3(c)-3(e) show the spin bias $V_{s}$ as a function of $I_{c}$ and $\gamma$. Later we will see in Secs. IV B and IV C that $I^{G}$ is ignorably small, thus hardly changing $V_{s}$. Thus, we let $I^{G}$ $=0$. As we see, $V_{s}$ can be as large as $\sim 0.1 \mathrm{meV}$ for the present device and parameters. $V_{s}$ increases linearly with $I_{c}$, while logarithmically with $\gamma . V_{s}$ changes sign with the current $I_{c}$

In the following, for a conservative and realistic simulation, we will always choose a relatively low injection efficiency $\gamma=0.1$ and assume $I^{G}=0$. Since the above boundary conditions only consider the conservation of current density, our results are valid when assuming the identical crosssection area in each part of the setup. One should consider different cross-section area in different regions and current conservation for a more general case. ${ }^{42}$

\section{Many-body states of the ferromagnetic nanograin}

In this work, we will describe the ferromagnetic nanograin by using the minimal possible model ${ }^{45,46}$ proposed to describe the experiment transport spectra through cobalt nanograin. 8,9 This model was also adopted to discuss spinpolarized current-induced relaxation and spin torque in the ferromagnetic nanograin. ${ }^{16,17,44}$

A full and detailed description of this model can be found in Appendix B. Simply speaking, at low temperatures, the grain can be described by the many-body states $\left|N, S, S_{z}\right\rangle$, where $N$ is the total electron number inside the grain, $S$ and $S_{z}$ are the magnitude and the $z$ component of the total angular momentum $\mathbf{S}$ of the grain, respectively.

In the following, we will focus on two branches of states. The first branch is

$$
\left|N_{0}, S_{0}, S_{z}\right\rangle
$$

i.e., there are $N=N_{0}$ electrons inside the grain, and the magnitude of the total angular momentum of the grain is $S=S_{0}$. Besides, since $S_{z} \in\left[-S_{0}, S_{0}\right]$, there are $2 S_{0}+1$ states in this branch. The second branch is obtained by adding an extra electron to the minority band of the grain with respect to the first branch, so that the total electron number increases by 1 and the magnitude of the total angular momentum decreases by $1 / 2$. This branch is denoted as

$$
\left|N_{0}+1, S_{0}-1 / 2, S_{z}\right\rangle,
$$

where $S_{z} \in\left[-S_{0}+1 / 2, S_{0}-1 / 2\right]$; i.e., there are $2 S_{0}$ states in this branch.

We refer regions (4)-(6) as the two "leads" connected to the grain, one is from regions (4) and (5), the other is from region (6). By tuning the gate voltage $V_{g}$ and applying the spin bias $V_{s}$ (induced by $I_{c}$ ), the energies of the two branches presented in Eqs. (10) and (11) can be set to be nearly degenerate with respect to the chemical potentials $\mu_{\uparrow,}^{(4)}(L)$, $\mu_{\uparrow, \downarrow}^{(5)}(0)$, and $\mu^{(6)}$. In this situation, the electrons in lead $(4,5)$ and lead (6) can be exchanged with the grain. Then we can use the polarization of the exchanged electrons to detect and manipulate the magnetization of the ferromagnetic grain.

\section{Rate equations in the presence of spin bias}

The evolution of the many-body states of the grain by exchanging electrons with the weakly coupled lead $(4,5)$ and (6) is described by the Pauli rate equations. ${ }^{52-54}$ We only consider the sequential tunneling regime. Born approximation and Markoff approximation are applied, and $H_{\mathrm{T}}$ is treated by perturbation up to the second order. ${ }^{53}$ The rate equation can be expressed in a compact form,

$$
\partial_{t} P_{l}=\sum_{l^{\prime}} R_{l l^{\prime}} P_{l^{\prime}},
$$

where $0 \leq P_{l} \leq 1$ are the probability to find the state $l$ $\equiv\left|N, S, S_{z}\right\rangle$. The diagonal and off-diagonal terms of the coefficient matrix of the rate equations are, respectively,

$$
R_{l^{\prime} \neq l}=\sum_{\alpha \sigma i} R_{l^{\prime} \neq l}^{\alpha \sigma i}, \quad R_{l l}=-\sum_{l^{\prime} \neq l} R_{l^{\prime} l},
$$

where

$$
\begin{aligned}
R_{l^{\prime} \neq l}^{\alpha \uparrow i}= & \Gamma_{\alpha} \cos ^{2} \frac{\theta_{\alpha}}{2}\left[\left|\left\langle l^{\prime}\left|d_{i \uparrow}\right| l\right\rangle\right|^{2} f\left(E_{l}-E_{l^{\prime}}-\mu_{+}^{\alpha}\right)\right. \\
& \left.+\left|\left\langle l\left|d_{i \uparrow}\right| l^{\prime}\right\rangle\right|^{2} f\left(E_{l}-E_{l^{\prime}}+\mu_{+}^{\alpha}\right)\right] \\
& +\Gamma_{\alpha} \sin ^{2} \frac{\theta_{\alpha}}{2}\left[\left|\left\langle l^{\prime}\left|d_{i \uparrow}\right| l\right\rangle\right|^{2} f\left(E_{l}-E_{l^{\prime}}-\mu_{-}^{\alpha}\right)\right. \\
& \left.+\left|\left\langle l\left|d_{i \uparrow}\right| l^{\prime}\right\rangle\right|^{2} f\left(E_{l}-E_{l^{\prime}}+\mu_{-}^{\alpha}\right)\right],
\end{aligned}
$$

and one just replaces $\uparrow$ with $\downarrow$ and exchanges + and - to obtain $R_{l^{\prime} l}^{\alpha \downarrow i}$. Note that the Fermi distribution $f(x)$ $=1 /\left[\exp \left(x / k_{B} T\right)+1\right]$ is spin resolved. The parameter $\Gamma_{\alpha}$ $=2 \pi \Sigma_{k}\left|V_{k \alpha i}\right|^{2} \delta\left(\omega-\epsilon_{k \alpha}\right)$ represents the spin-irrelevant coupling between the lead $\alpha \in\{(4,5),(6)\}$ and the grain. For simplicity, we assume that $\Gamma_{(4,5)}=\Gamma_{(6)}=\Gamma$, and $\Gamma$ are assumed to be independent of the specific single-particle level $i$. The overlapping $\left\langle l\left|d_{i \sigma}\right| l^{\prime}\right\rangle$ can be found by calculating the Clebsch-Gordan coefficients.

$\theta_{\alpha}$ in Eq. (14) is the angle between the easy axis of grain and the spin-quantization direction of the lead $\alpha$. For simplicity, we set the easy axis of the grain as $z$ axis and assume that $\theta_{(6)}=0$ and $\theta_{(4,5)}=\theta \in[0, \pi / 2]$, as shown in Fig. 4. In the following, we denote $\mu_{+/-}^{(4)}=\mu_{\uparrow / \downarrow}^{(4)}$ when $\theta=0$.

In terms of $P_{l}$, the magnetization of the grain is given by 


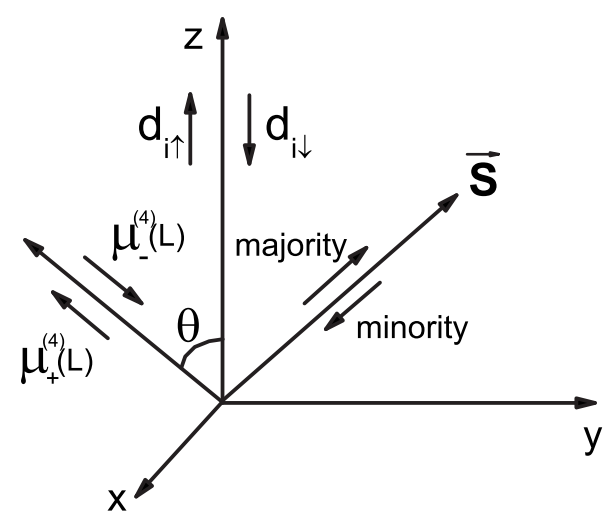

FIG. 4. The easy axis of the grain is set as $z$ direction. The grain electrons $\left(d_{i \uparrow / \downarrow}\right)$ are quantized along $z$ direction. The majority and minority electrons orient parallel and antiparallel with $\mathbf{S}$, respectively. $\mathbf{S}$ is the total angular momentum of the grain formed by all the electrons inside the grain. $\theta$ is the angle between the easy axis of grain and the spin-quantization direction of lead $(4,5)$.

$$
M=\sum_{l} S_{z}^{l} P_{l},
$$

and the spin- $\sigma$ current flowing from the region $\alpha$ into the grain are defined as

$$
I_{\alpha}^{\sigma}=-e \sum_{l l^{\prime}}\left(N_{l}-N_{l^{\prime}}\right) R_{l^{\prime} l}^{\alpha \sigma} P_{l^{\prime}},
$$

where $N_{l}$ and $S_{z}^{l}$ correspond to the $N$ and $S_{z}$ of the state $l$ $\equiv\left|N, S, S_{z}\right\rangle$.

The validity of the rate equations is discussed in Appen$\operatorname{dix}$ C.

\section{NLSV SIGNAL IN THE TUNNELING REGIME}

In this section, we will present the microscopic picture of the NLSV signal in the sequential tunneling regime. When a small current $I_{c}$ is driven in the loop formed by regions (1)(3) of Fig. 1(a), the relative alignment of magnetization between the two ferromagnets can be read out by measuring the voltage difference between regions (6) and (5).

\section{A. $\theta=0$ case}

We will discuss first the case when $\theta=0$. For $\theta=0$, we denote $\mu_{+/-}^{(4)}=\mu_{\uparrow / l}^{(4)}$. The results can be easily generalized for $\theta \neq 0$ in Sec. III B. The left and right columns of Fig. 5 show the cases when the magnetization of the grain is antiparallel $(\mathrm{AP})$ and parallel $(P)$ with injector $(1)$, respectively. First, we consider the AP case, i.e., $S_{z}$ of the grain $\sim-S_{0}$. By putting the experimental measurement current ${ }^{37} I_{c}=250 \mu \mathrm{A}$ into Eq. (8) and assuming $\gamma=0.1$ and $I^{G}=0$, we estimate that a positive spin bias $V_{s} \sim 1.84 \mu \mathrm{eV}$ will be induced at the interface where regions (4), (5) and the grain connect, so that $\mu_{\uparrow / \downarrow}^{(4)}(L)=\mu_{\uparrow / \downarrow}^{(5)}(0)= \pm V_{s} / 2$. It is natural to assume that the distance between the voltmeter and the (4)/(5)/grain interface $\gg \lambda_{N}=1 \mu \mathrm{m}$, so the split chemical potentials for spin-up and -down electrons will decay to only one chemical potential $\mu^{(5)}(\infty)$ on the voltmeter side of region (5). Because region
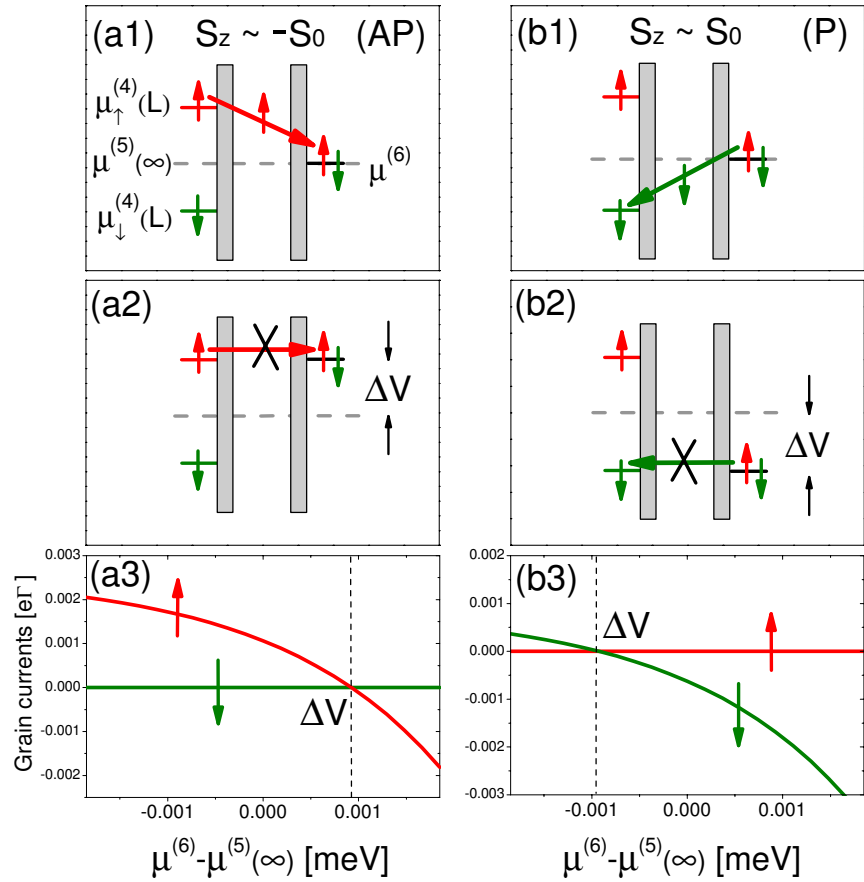

FIG. 5. (Color online) Using NLSV signal in the sequential tunneling regime to read out the magnetization of the grain. (a1) (a3) and (b1)-(b3) show the cases when the magnetization of the grain is AP and $P$ with injector (1), respectively. $\mu^{(5)}(\infty)$ and $\mu^{(6)}$ are the chemical potentials on the voltmeter side of regions (5) and (6), respectively. (a1) When $S_{z} \sim-S_{0}$, the grain favors spin-up current flowing from lead $(4,5)$ to lead $(6)$. Because it is an open circuit between lead $(4,5)$ and lead (6), the electrons flowing into lead (6) cannot go anywhere but build up and raise the chemical potential $\mu^{(6)}$. (a2) Once the chemical potential $\mu^{(6)}$ is raised to be aligned with $\mu_{\uparrow}^{(4)}(L)$, the current flowing and electron building-up stop. Then one can measure a voltage difference between $\mu^{(5)}(\infty)$ and $\mu^{(6)}$, which corresponds to the NLSV voltage. (a3) The spin-up and -down currents as functions of $\mu^{(6)}-\mu^{(5)}(\infty)$. The NLSV voltage $\Delta V=\mu^{(6)}-\mu^{(5)}(\infty)$ corresponds to the vertical dashed line at which both currents are zero. (b1)-(b3) can be understood similarly. The parameters: $\theta=0, I_{c}=250 \mu \mathrm{A}, \gamma=0.1, I^{G}=0, S_{0}=100, T=20 \mathrm{mK}$, $\Delta V_{g}=-K_{N}$, and other parameters are the same as those in Fig. 3 .

(5) is nonmagnetic, the decays of spin-up and -down chemical potentials are symmetric, so it can be anticipated that $\mu^{(5)}(\infty)=\left[\mu_{\uparrow}^{(4)}(L)+\mu_{\downarrow}^{(4)}(L)\right] / 2=\left[\mu_{\uparrow}^{(5)}(0)+\mu_{\downarrow}^{(5)}(0)\right] / 2$. This situation is depicted by $\mu_{\uparrow / \downarrow}^{(4)}(L)$ and $\mu^{(5)}(\infty)$ in Fig. 5(a1).

In the response of this small spin bias, a small current will be generated, flowing through the grain. Specifically, spin-up current will be favored for the AP case. This can be understood with the help of the Clebsch-Gordan coefficient,

$$
\begin{aligned}
& \left\langle N_{0}, S_{0}, S_{z}\left|d_{i \uparrow}\right| N_{0}+1, S_{0}-\frac{1}{2}, S_{z}+\frac{1}{2}\right\rangle \\
& \quad=\left\langle j_{1}=S_{0}, m_{1}=S_{z} ; j_{2}=\frac{1}{2}, m_{2}=\frac{1}{2} \mid J=S_{0}-\frac{1}{2}, M=S_{z}+\frac{1}{2}\right\rangle \\
& \quad=-\sqrt{\frac{S_{0}-S_{z}}{2 S_{0}+1}},
\end{aligned}
$$




$$
\begin{aligned}
& \left\langle N_{0}, S_{0}, S_{z}\left|d_{i \downarrow}\right| N_{0}+1, S_{0}-\frac{1}{2}, S_{z}-\frac{1}{2}\right\rangle \\
& \quad=\left\langle j_{1}=S_{0}, m_{1}=S_{z} ; j_{2}=\frac{1}{2}, m_{2}=-\frac{1}{2} \mid J=S_{0}-\frac{1}{2}, M=S_{z}-\frac{1}{2}\right\rangle \\
& \quad=\sqrt{\frac{S_{0}+S_{z}}{2 S_{0}+1}}
\end{aligned}
$$

apparently, for $S_{z} \sim-S_{0}$, the probability for spin-up electrons to tunnel through the grain,

$$
\frac{S_{0}-\left(-S_{0}\right)}{2 S_{0}+1} \sim 1,
$$

is much larger than the probability for spin-down electrons

$$
\frac{S_{0}+\left(-S_{0}\right)}{2 S_{0}+1} \sim 0 .
$$

In other words, the spin-down current is magnetic blockaded. ${ }^{16}$ Note that the spin selection rules remain qualitatively unchanged even for small fluctuation of $S_{z}$ around $-S_{0}$, as long as $S_{0} \gg 1$.

As a result, the favored spin-up electrons will flow from region (4) through the grain into region (6), if $\mu_{\uparrow}^{(4)}(L)$ $>\mu^{(6)}$. Remember that there is a voltmeter between regions (5) and (6), so the electrons tunneling into region (6) cannot go anywhere but accumulate and raise the chemical potential of region (6) until $\mu^{(6)} \sim \mu_{\uparrow}^{(4)}(L)$. After this accumulation is accomplished, no more current will flow and there is finally a stable voltage difference $\Delta V$ between $\mu^{(6)}$ and $\mu^{(5)}(\infty)$, as shown in Fig. 5(a2).

Figure 5(a3) shows how to numerically determine the NLSV voltage $\Delta V$. One just scan $\mu^{(6)}$ and calculate the tunneling current through the grain. $\Delta V$ is then found out as at which $\mu^{(6)}-\mu^{(5)}(\infty)$ both the spin-up and -down currents vanish, as indicated by the vertical dashed line. It turns out that in the sequential tunneling regime and when $\theta=0$, the magnitude of the NLSV voltage is

$$
\left|\Delta V^{\theta=0}\right|=\left|V_{s}\right| / 2 .
$$

By using Eq. (8) and the parameters given in Fig. 5, for $I_{c}$ $=250 \mu \mathrm{A}$, the NLSV voltage $\Delta V^{\theta=0}$ is found out to be about $0.92 \mu \mathrm{V}$. The NLSV signal is thereby equal to

$$
R_{\mathrm{NLSV}}^{\theta=0} \equiv \frac{\Delta V^{\theta=0}}{I_{c}}=\frac{0.92 \mu \mathrm{V}}{250 \mu \mathrm{A}} \simeq 3.7 \mathrm{~m} \Omega .
$$

Similarly, as shown in Figs. 5(b1)-5(b3), the parallel $(P)$ case favors that spin-down current flowing from region (6) through the grain to regions (4) and (5) also due to the same spin selection rules Eq. (17). This current will drain the electrons in region (6) and lower $\mu^{(6)}$ until $\mu^{(6)} \sim \mu_{\downarrow}^{(4)}(L)$. Therefore, for the $P$ case, $\Delta V^{\theta=0}=-0.92 \mu \mathrm{eV}$ and $R_{\mathrm{NLSV}}^{\theta=0}=$ $-3.7 \mathrm{~m} \Omega$, right opposite to the AP case.

\section{B. $\theta \neq 0$ case}

When $\theta \neq 0$, the chemical potentials of lead $(4,5)$ now are denoted as $\mu_{+}^{(4)}(L)$ and $\mu_{-}^{(4)}(L)$, respectively. We still take $S_{z} \sim-S_{0}$ for example. As shown in Fig. 6(a), when $\theta \neq 0$, the favored spin- $\uparrow$ current can flow not only from $\mu_{+}^{(4)}(L)$ to $\mu^{(6)}$,
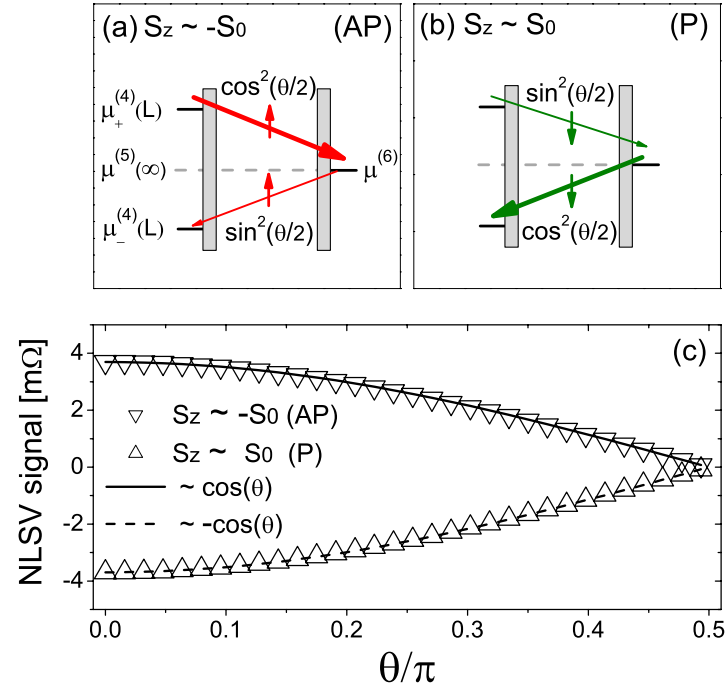

FIG. 6. (Color online) NLSV signal when $\theta \neq 0$. (a) when $\theta$ $\neq 0$ and the magnetization of the grain is $S_{z}=-S_{0}$ (AP), the spin-up current can flow simultaneously from lead $(4,5)$ to lead (6) with relative probability $\cos ^{2} \frac{\theta}{2}$, and from lead (6) to lead (4,5) with probability $\sin ^{2} \frac{\theta}{2}$. (b) when $\theta \neq 0$ and the magnetization of the grain is $S_{z}=S_{0}(\mathrm{p})$, spin-down current can flow simultaneously from lead $(4,5)$ to lead (6) with relative probability $\sin ^{2} \frac{\theta}{2}$, and from lead (6) to lead $(4,5)$ with probability $\cos ^{2} \frac{\theta}{2}$. (c) The NLSV signal as a function of $\theta$ for the $\mathrm{P}(\triangle)$ and AP $(\nabla)$ cases. $\pm \cos \theta$ are also plotted for comparison. Other parameters are the same as Fig. 5.

but also from $\mu^{(6)}$ to $\mu_{-}^{(4)}(L)$, according to the rate equations Eq. (14). These two currents are denoted as $I_{\mu_{+}^{(4)}(L) \rightarrow \mu^{(6)}}^{\uparrow}$ and $I_{\mu^{(6)} \rightarrow \mu_{-}^{(4)}(L)}^{\uparrow}$, respectively.

According to the rate coefficients [Eq. (14)], the spin-up electrons are related to $\mu_{+}^{(4)}(L)$ by $\cos ^{2} \frac{\theta}{2}$, and to $\mu_{-}^{(4)}(L)$ by $\sin ^{2} \frac{\theta}{2}$. Besides, lead (6) is not spin dependent, so the polarization and magnitude of the current between the grain and (6) just follow those between (4) and the grain. Therefore,

$$
\begin{aligned}
& I_{\mu_{+}^{(4)}(L) \rightarrow \mu^{(6)}}^{\uparrow} \propto \cos ^{2} \frac{\theta}{2}, \\
& I_{\mu^{(6) \rightarrow \mu_{-}^{(4)}(L)}}^{\uparrow} \propto \sin ^{2} \frac{\theta}{2},
\end{aligned}
$$

respectively. As a result, $\mu^{(6)}$ will saturate to a balanced position at which the above two opposite currents cancel with each other. Because $\cos ^{2} \frac{\theta}{2}-\sin ^{2} \frac{\theta}{2}=\cos \theta$, this balanced position of $\mu^{(6)}$ turns out to be proportional to $\cos \theta .^{55}$

The triangles in Fig. 6(c) show the self-consistent results of the NLSV signal as a function of $\theta$. For comparison, the functions $\pm \cos \theta$ are also plotted by solid and dashed lines, respectively. One easily concludes that the NLSV signal in the presence of $\theta$ is given by

$$
R_{\mathrm{NLSV}}= \pm \frac{V_{s}}{2 I_{c}} \cos \theta
$$

where \pm depends on the magnetization of the grain. 
TABLE I. Parameters for a $S_{0}=1000$ grain. All energies are in meV (Refs. 45 and 61). $E_{C}, S_{0}, K_{N}$ are estimated by experiments (Refs. 8-11) $\delta_{a}, \delta_{i}$, and $\Delta_{F}^{S_{0}}$ are from the band calculations. ${ }^{62}$ According to Eq. (B5), $\bar{E}_{F}^{S_{0}}$ can be absorbed into $V_{g}$, thus is set to 0 for convenience. $\mu^{(5)}(\infty)$ is the energy zero point throughout the paper.

\begin{tabular}{ccccccc}
\hline \hline$E_{C}$ & $K_{N}$ & $\delta_{a}$ & $\delta_{i}$ & $\Delta_{F}^{S_{0}}$ & $\bar{E}_{F}^{S_{0}}$ & $\mu^{(5)}(\infty)$ \\
\hline 30 & 0.01 & 4.61 & 1.19 & 2000 & 0 & 0 \\
\hline \hline
\end{tabular}

\section{Discussion}

There are several points should be clarified:

(i) $R_{\mathrm{NLSV}}$ we obtained for the present device and parameters happen to be of the same order as the experimental observations, where the NLSV signals are of the order of 1 $\sim 10 \mathrm{~m} \Omega$. $^{37}$

(ii) Above, we only consider the case that $S$ decreases by $1 / 2$ when the extra electron is added, where $S$ is the magnitude of the total angular momentum of the grain. Also, according to Fig. 11, there is small probability that $S$ increases by $1 / 2$ when adding the extra electron, i.e., the extra electron is preferred to be added to the majority band, then the spin selection rules will become totally reversed as

$$
\begin{gathered}
\left\langle N_{0}, S_{0}, S_{z}\left|d_{i \uparrow}\right| N_{0}+1, S_{0}+\frac{1}{2}, S_{z}+\frac{1}{2}\right\rangle=\sqrt{\frac{S_{0}+S_{z}+1}{2 S_{0}+1}}, \\
\left\langle N_{0}, S_{0}, S_{z}\left|d_{i \downarrow}\right| N_{0}+1, S_{0}+\frac{1}{2}, S_{z}-\frac{1}{2}\right\rangle=\sqrt{\frac{S_{0}-S_{z}+1}{2 S_{0}+1}}
\end{gathered}
$$

so that tunneling of spin-up (spin-down) electrons will be favored when $S_{z} \sim S_{0}\left(S_{z} \sim-S_{0}\right)$. Therefore, the results will be totally reversed. This is a direct consequence of the strong Coulomb repulsion and the unequal spacings $\delta_{a}$ and $\delta_{i}$ of majority and minority one-particle levels for an ultrasmall grain, and a major difference from the relatively large films. ${ }^{36,37}$

(iii) Note that in regions (4) and (5), the steady-state splitting of spin-up and -down chemical potentials is maintained by the spins continuously injected by $I_{c}$. This is different from in region (6), where there should be no current flowing in or out at steady state, because of the voltmeter. Therefore, in region (6), the spin-up and -down electrons will eventually relax to one chemical potential for sufficient long time. That is why we consider, for the steady-state solution, only one spin-irrelevant chemical potential $\mu^{(6)}$ in region (6).

(iv) In the simulation, although the parameters listed in Table I for a $S_{0}=1000$ grain are exploited, we only use $S_{0}$ $=100$ for simulation because of the limited computing power. We have checked the results from $S_{0}=10$ through $S_{0}=100$, and the results turn out to be quantitatively unchanged as long as $S \gg 1$.

\section{MAGNETIZATION SWITCHING}

In this section, we will present the magnetization switching of the grain under a current $I_{c}$ exceeding a critical value.
This critical $I_{c}$ is determined by the gate voltage $V_{g}$ and the spin bias $V_{s}$, at which the strong Coulomb and magnetic blockades of the grain are lifted. Then currents can flow through the grain, and transfer the angular momentum carried by the flowing electrons to the grain. This will be discussed in Sec. IV A.

Besides, still because of these blockades, one cannot preassume there is always a pure spin current flowing through the grain, so whether the reversal is accompanied by the pure spin current is waited to be checked. This will be discussed in Sec. IV B.

\section{A. Critical $I_{c}$ and $V_{s}$}

As we have discussed, the electron tunneling between the lead and the grain are subjected to the Coulomb and magnetic blockades simultaneously. According to Eq. (B12), by choosing suitable gate voltage, the charging energy $E_{C}$ can be compensated, but the transition energies still depend on the magnetization of the grain; i.e., the grain may be magnetic blockaded. ${ }^{16}$ In this situation, this magnetic blockade can be lifted by applying the spin bias $V_{s}$ exceeding a critical value, which thereby defines the minimal critical $I_{c}$ and $V_{s}$. We will use the reversal from $S_{z}=-S_{0}$ to $S_{0}$ to extract the minimal critical $I_{c}$ and $V_{s}$ at which the magnetic blockade can be lifted and the switching can be performed.

Suppose the grain is initially prepared at the state $\left|N_{0}, S_{0},-S_{0}\right\rangle$. By adding a spin-up electron from lead $(4,5)$ into the minority band of the grain, the grain will transit to the state $\left|N_{0}+1, S_{0}-\frac{1}{2},-S_{0}+\frac{1}{2}\right\rangle$. This transition, energetically requires that

$$
\left.\mu_{\uparrow}^{(4)}(L)>E_{\left|N_{0}+1, S_{0}-1 / 2,-S_{0}+1 / 2\right\rangle}-E_{\left|N_{0}, S_{0},-S_{0}\right\rangle}\right\rangle
$$

Via this transition, $S_{z}$ increases by $1 / 2$ unit.

Further, by draining a spin-down electron from the minority band of the grain to lead $(4,5)$, the grain will transit from the state $\left|N_{0}+1, S_{0}-\frac{1}{2},-S_{0}+\frac{1}{2}\right\rangle$ to $\left|N_{0}, S_{0},-S_{0}+1\right\rangle$. This energetically requires that

$$
\mu_{\downarrow}^{(4)}(L)<E_{\left|N_{0}+1, S_{0}-1 / 2,-S_{0}+1 / 2\right\rangle}-E_{\left|N_{0}, S_{0},-S_{0}+1\right\rangle} .
$$

Via this transition, $S_{z}$ also increases by $1 / 2$ unit.

Note that $\mu_{\uparrow / \downarrow}^{(4)}(L)= \pm V_{s} / 2$. If one applied a sufficient large $I_{c}$, so that the spin bias $V_{s}$ driven by $I_{c}$ is large enough, for all the possible $S_{z} \in\left[-S_{0}, S_{0}\right]$, there are always

$$
\mu_{\downarrow}^{(4)}(L)<\left\{E_{\left|N_{0}+1, S_{0}-1 / 2, S_{z} \pm 1 / 2\right\rangle}-E_{\left|N_{0}, S_{0}, S_{z}\right\rangle}\right\}<\mu_{\uparrow}^{(4)}(L) .
$$

Then, one can expect a sequence of consecutive chargingdischarging steps to be driven, which charges the grain with 

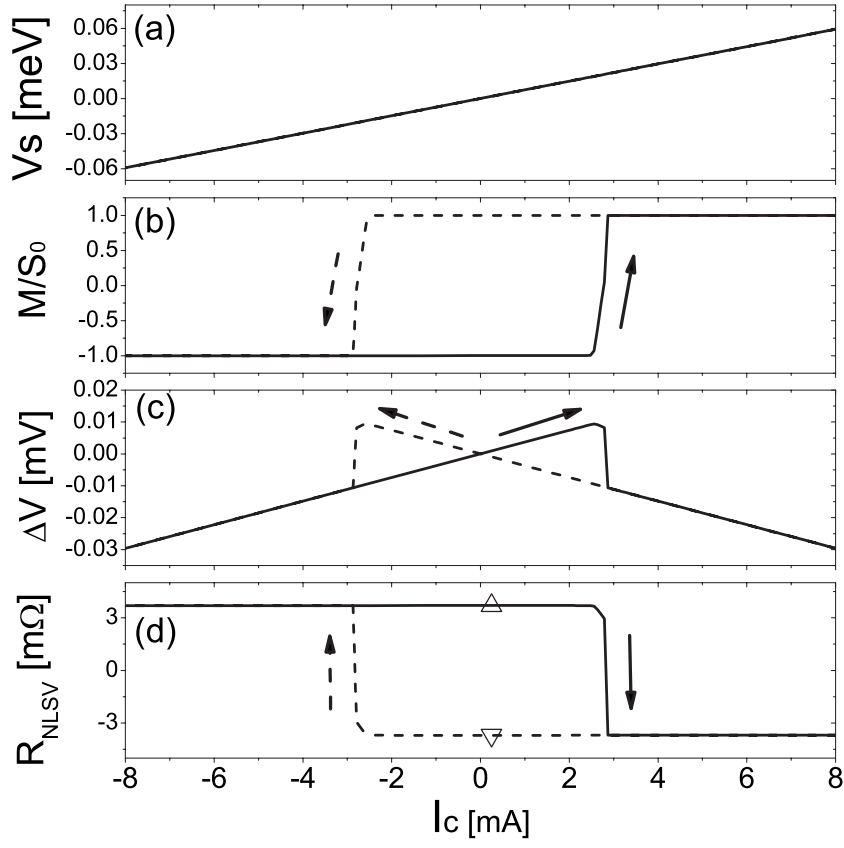

FIG. 7. (a) The spin bias $V_{s}$, (b) the normalized magnetization, (c) the NLSV voltage, and (d) the NLSV signal as functions of the driving current $I_{c}$. The parameters $\theta=0, \gamma=0.1, I^{G}=0, S_{0}=100, T$ $=20 \mathrm{mK}, \Delta V_{g}=0$, and other parameters are the same as those in Fig. 3.

only spin-up electrons and discharges the grain with only spin-down electrons. As a result of this charging-discharging sequence, the magnetization of the grain will eventually be reversed from $S_{z}=-S_{0}$ to $S_{0}$.

Similarly, to reverse the magnetization from $S_{z}=S_{0}$ to $-S_{0}$, the energy requirement is that for all the possible $S_{z}$,

$$
\mu_{\uparrow}^{(4)}(L)<\left\{E_{\left|N_{0}+1, S_{0}-1 / 2, S_{z} \pm 1 / 2\right\rangle}-E_{\mid N_{0}, S_{0}, S_{z}}\right\}<\mu_{\downarrow}^{(4)}(L) .
$$

Therefore, the minimal required $V_{s}$, which equals $\mu_{\uparrow}^{(4)}(L)-\mu_{\downarrow}^{(4)}(L)$, is determined by the width of spectrum $E_{\left|N_{0}+1, S_{0}-1 / 2, S_{z} \pm 1 / 2\right\rangle}-E_{\left|N_{0}, S_{0}, S_{z}\right\rangle}$ for all $S_{z} \in\left[-S_{0}, S_{0}\right]$. According to Eq. (B12), this spectrum width is $2 K_{N}$, which thereby set the value for the minimal required spin bias. The minimal critical current is then defined as the $I_{c}$ by which the generated $V_{s} \geq 2 K_{N}$.

Figure 7 shows $V_{s}$, magnetization, and NLSV signals as functions of $I_{c}$, when $\theta=0$. For each $I_{c}, V_{s}$ is calculated first using Eq. (8). Then, $V_{s}$ is put into the rate equations [Eq. (12)] to self-consistently determine $\Delta V$ and $\mu^{(6)}$ by using the same method shown in Figs. 5(a3) and 5(b3) until both spin-up and -down currents through the grain vanish. Finally, the magnetization is obtained by putting the calculated $\mu^{(6)}$ back to the rate equations. The NLSV signal $R_{\mathrm{NLSV}}$ is found by $R_{\mathrm{NLSV}}=\Delta V / I_{c}$. These steps is shown by Fig. 2 .

The triangles $\nabla$ and $\Delta$ in Fig. 7 indicate the $P$ and AP cases we have already discussed in Fig. 5, respectively. Keep increasing $I_{c}$ until $V_{s}$ exceeds $2 K_{N}$, the magnetization of the grain will be reversed. In the present set of parameters, the steady-state magnetization starts to reverse when $\left|I_{c}\right|$ is a little larger than $2.5 \mathrm{~mA}$. The switching is accomplished after $\left|I_{c}\right|$ exceeds $\sim 3 \mathrm{~mA}$, at which $\left|V_{s}\right|$ is right larger than $2 K_{N}$ $=0.02 \mathrm{meV}$. We attribute the broadening of reversal point at $\left|I_{c}\right|=2.5 \sim 3 \mathrm{~mA}$ to the thermal fluctuation of the lead electron bath. At the reversal point, both the sign and slope of $\Delta V$ changes abruptly. $R_{\mathrm{NLSV}}$ also demonstrates a hysteresis loop in analogy to the hysteresis loop of the magnetization but with opposite signs. This opposition has already been explained in the discussion (ii) of Sec. III.

There are several points needed to be clarified:

(i) the results should be qualitatively unaffected for small $\theta \neq 0$ because $\theta$ does not change $E_{\left|N_{0}+1, S_{0}-1 / 2, S_{z} \pm 1 / 2\right\rangle}$ $-E_{\left|N_{0}, S_{0}, S_{z}\right\rangle}$, while only these energy differences determine the critical spin bias $V_{s}$ and $I_{c}$;

(ii) we have concluded that the minimal critical current is only related to $K_{N}$, which does not depend on $S_{0}$, so we use $S_{0}=100$ to perform the calculation. We have checked that the simulation results for other $S_{0} \gg 1$ turn out to be qualitatively unchanged.

(iii) According to Eq. (B9), $E_{\left|N_{0}+1, S_{0}-1 / 2, S_{z} \pm 1 / 2\right\rangle}-E_{\left|N_{0}, S_{0}, S_{z}\right\rangle}$ is also a function of the gate voltage $V_{g}$; therefore, the critical $I_{c}$ can be tuned by the gate voltage. Above we only discuss the minimal critical $I_{c}$; i.e., the case when the Coulomb and the one-particle energies are already compensated by choosing suitable gate voltage (details can be found in Appendix B 5). Therefore, the spin bias only has to lift the magnetic blockade and thereby can be minimized. If the nearly degenerate situation were tuned away by a magnitude of $\Delta V_{g}$, the spin bias $V_{s}$ then has to compensate the Coulomb and magnetic blockades simultaneously. Then, the critical $V_{s}$ will become $\left|2 K_{N}\right|+\left|\Delta V_{g}\right|$, which thereby requires larger critical $I_{c}$.

(iv) The critical current density. For the present parameters, the critical current is about $I_{c}=3 \mathrm{~mA}$, while the crosssection area is $A_{\square}=170 \times 65 \mathrm{~nm}^{2}$. Therefore, the critical current density is about

$$
\frac{I_{c}}{A_{\square}}=\frac{3 \times 10^{-3} \mathrm{~A}}{170 \times 10^{-7} \mathrm{~cm} \times 65 \times 10^{-7} \mathrm{~cm}} \approx 2.7 \times 10^{7} \mathrm{~A} / \mathrm{cm}^{2}
$$

This value is comparable to most experiments of nanopillars and multilayers. ${ }^{26}$

\section{B. Pure spin current}

We are particularly interested in whether there is truly a pure current flowing through the grain during the reversal in the present device. Therefore, we studied a situation that the grain is initialized at $S_{z}=-S_{0}$ and $I_{c}$ is suddenly switched on to generate a spin bias large enough to drive a switching from $S_{z}=-S_{0}$ to $S_{0}$, then see how the spin-resolved currents and the magnetization evolve with time.

Roughly speaking, if we assume the electrons tunneling through the grain transfer all their angular momenta to the grain, to reverse the grain from $S_{z}=-S_{0}$ to $S_{0}$, there should be at least $4 S_{0}$ electrons tunneling through the grain during the reversal. Because the grain is much smaller than region (6), it is safe to expect that the electrons flowing in and out region (6) along with the reversal process will hardly change $\mu^{(6)}$. 


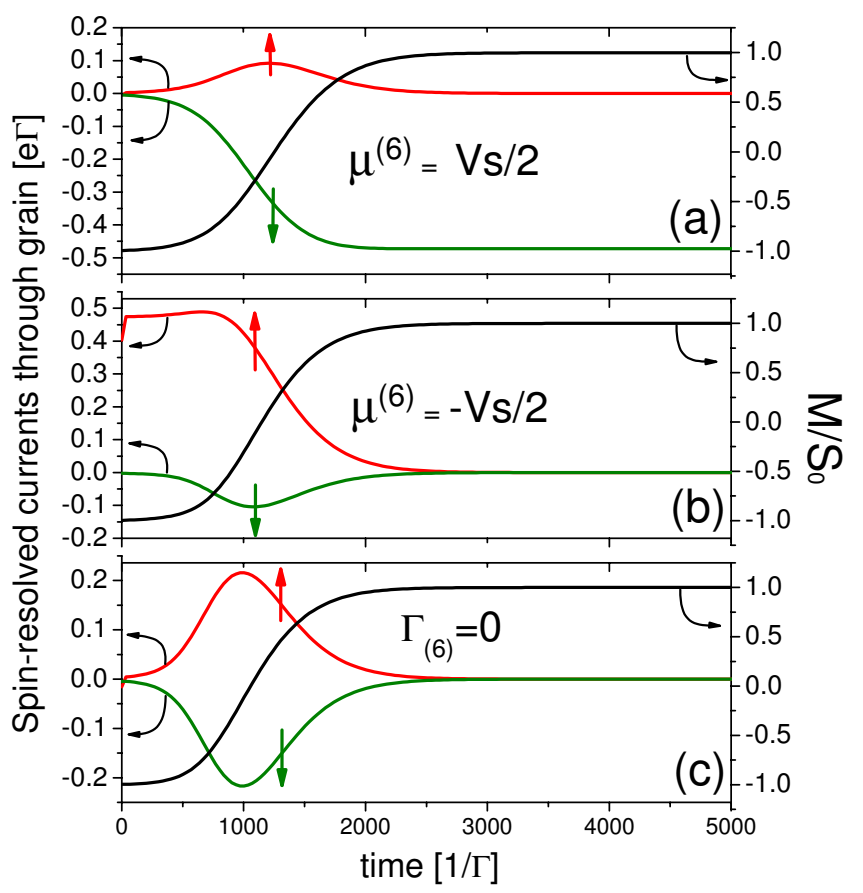

FIG. 8. (Color online) The spin-up and -down currents flowing through the grain and the magnetization of the grain as functions of time when assuming that the chemical potential of region (6) is: (a) $\mu^{(6)}=V_{s} / 2$ and (b) $\mu^{(6)}=-V_{s} / 2$. (c) region (6) is not connected to the grain. The positive direction of current is defined as flowing from lead (4,5) to lead (6). $\theta=0, V_{s}=3 K_{N}, \gamma=0.1, T=20 \mathrm{mK}, S_{0}$ $=100, \Delta V_{g}=0$, and other parameters are the same as those in Fig. 3 .

According to Fig. 5(a2), when $V_{s}$ is positive while $S_{z}=$ $-S_{0}, \mu^{(6)}$ will saturate at $V_{s} / 2$; and when $V_{s}>0$ and $S_{z}=S_{0}$, $\mu^{(6)}$ will saturate at $-V_{s} / 2$. So in the following we will compare two limits. The first limit assumes that $\mu^{(6)}=V_{s} / 2$. The second assumes that $\mu^{(6)}=-V_{s} / 2$. These two limits are shown in Figs. 8(a) and 8(b), respectively.

Let us first consider the first limit $\mu^{(6)}=V_{s} / 2$ $=0.015 \mathrm{meV}$. This situation is the same as shown in Fig. 5 (a2), but $V_{s}$ is much larger in magnitude. According to Fig. $8(\mathrm{a})$, as the magnetization changes from $-S_{0}$ to $S_{0}$, the spindown current gradually becomes favored and the spin-up current unfavored, which is consistent with the spin selection rules [Eq. (17)]. Remember the configuration of chemical potentials remains unchanged during the reversal as shown in Fig. 5(a2). As a result, the magnitude of spin-down current will keep growing during the reversal, and even after the magnetization is reversed to $S_{0}$, there will still be a steady spin-down current flowing from lead (6) to lead $(4,5)$. Although not shown in our result, one can expect, for sufficient long time, the spin-down current leaking from region (6) will eventually shift $\mu^{(6)}$ down to $-V_{s} / 2$, much like the same situation as shown by Fig. 5(b2). Then the spin-down current will cease to flow.

The second limit is shown by Fig. 8(b). At the start, $S_{z}=$ $-S_{0}$, so spin-up current is favored to flow from lead $(4,5)$ to lead (6). As the reversal from $-S_{0}$ to $S_{0}$ goes on, the spin-up current will gradually become unfavored, and drop to zero after the reversal is accomplished. Although the spin-down current is favored when $S_{z}=S_{0}$, the Fermi levels for spin- down electrons on both sides of the grain are the same, so neither the spin-up nor -down current will be flowing after the reversal.

After considering the above two limits, it is natural to expect that a real situation should be between them, i.e., $\mu^{(6)}$ should float gradually from $V_{s} / 2$ to $-V_{s} / 2$, and there should be a spin-polarized current instead of the anticipated pure spin current flowing through the grain during and after the reversal, until the accumulation or drainage in floating region (6) is accomplished.

The extra charge part of the current is produced along with the electron accumulation or drainage process in region (6). To prove this point, we decouple region (6) completely by letting $\Gamma_{(6)}=0 .{ }^{21}$ The results are shown in Fig. $8(\mathrm{c})$. In this case, the spin-up and -down currents are always the same in magnitude and opposite in direction, i.e., there is only a pure spin current flowing. Besides, by integrating the current over time using the data of Fig. 8(c),

$$
Q_{\uparrow / \downarrow}=\int_{0}^{\infty} I_{(4)(5)}^{\uparrow \downarrow} d t
$$

we obtain that both the spin-up electrons that tunnel into the grain and spin-down electrons off the grain are $2 S_{0}$. This is consistent with the change of $S_{z}$ by $2 S_{0}$ during the reversal, where half is from the $2 S_{0}$ incoming spin-up electrons and half the $2 S_{0}$ outgoing spin-down electrons.

\section{Validity of approximation $I^{G}=0$}

Because surrounded by insulator, the grain is connected to the leads via the quantum tunneling. Ref. 20 of Ref. 46 estimates that the tunneling rate for Ref. 8 is around $\Gamma$ $=10^{9} \mathrm{~s}^{-1}$. According to Fig. 7, where either the magnitude of spin-up or -down current is smaller than $e \Gamma$. Therefore, we estimate the current in and out the grain $I^{G}$ are well smaller than

$$
e \Gamma=1.6 \times 10^{-19} \text { Coulomb } \times 10^{9} s^{-1}=1.6 \times 10^{-10} \mathrm{~A}
$$

This value is 6-7 orders smaller than, e.g., the driven current $I_{c}\left(\sim 10^{-3} \mathrm{~A}\right)$ shown in our Fig. 7. So it is valid for us to ignore $I^{G}$ in our numerical calculations, though we explicitly kept it in Eq. (8).

\section{SUMMARY}

In this work, we theoretically studied the magnetization switching and detection of a ferromagnetic nanograin in a nonlocal spin valve (NLSV) device. Different from the original experiment, ${ }^{37}$ our nanograin is much smaller in size and at much lower temperatures, thus subjected to strong Coulomb and magnetic blockades. We describe the grain as a Stoner particle, whose ferromagnetism comes from the exchange interactions between itinerant electrons inside it. Because of the ultrasmall size, the one-particle levels inside it are quantized. Because of the ferromagnetism, the level spacings for the majority and minority electrons are unequal. 
As shown in Fig. 1(a), the grain is coupled to regions (4)-(6) of the NLSV device via quantum tunneling. Regions (4) and (5), and region (6) can be regarded as two nonmagnetic leads, respectively. In the lead formed by regions (4) and (5), a spin-dependent splitting of chemical potentials (spin bias) is induced by the spin-polarized current $I_{c}$ injected from ferromagnetic injector (1), as shown in Figs. 3(a) and 3(b). The other electrode (6) is floating and connected to region (5) through a voltmeter.

By applying a $I_{c}$ and measure the voltage difference $\Delta V$ between regions (5) and (6), the magnetization of the grain can be read out by the NLSV signal $R_{\mathrm{NLSV}}=\Delta V / I_{c}$. Because of the unequal level spacings for the majority and minority electrons and Coulomb blockade, the NLSV signal in the tunneling regime depends not only on the magnetization of the grain, but also on whether the majority or minority band of the grain is favored to contribute to the electron transport. The results when the minority band is favored are right opposite to when the majority band is favored. In the presence of an angle $\theta$ between the easy axis of the grain and the spin-quantization direction of the electrode, the NLSV signal is proportional to $\cos \theta$ and vanishes when $\theta=\pi / 2$.

By applying $I_{c}$ exceeding a critical value, the magnetization of the grain can be switched reversibly by the spin bias generated by the $I_{c}$. Because of the strong Coulomb and magnetic blockades, the electron flowing between the grain and the electrodes is not possible unless both blockades are lifted, then the angular momenta carried by the flowing electrons can be transferred to the grain. Therefore, the critical value of $I_{c}$ to drive the magnetization switching is determined by: at what gate voltage $V_{g}$ and spin bias $V_{s}$, both the Coulomb and magnetic blockades can be lifted. We also show that the current accompanying the switching may not be a pure spin current, due to the accumulation or drainage of electrons in the floating lead used for the NLSV measurement. A possible solution is to remove the floating lead.

Our numerical evaluations using realistic parameters from the recent NLSV (Refs. 36 and 37) and the cobalt grain experiments ${ }^{8-11}$ show that it is possible to employ the NLSV device to detect and switch the magnetization of a ferromagnetic nanograin under the present experimental conditions.

\section{ACKNOWLEDGMENTS}

We thank Wei-Qiang Chen, Zhong-Yi Lu, Rong Lv, ChaoXing Liu, Zhan-Feng Jiang, Rui-Lin Chu, and Wen-Yu Shan for helpful discussions. This work was supported by the Research Grant Council of Hong Kong under Grants No. HKU 704809 and No. HKU 10/CRF/08.

\section{APPENDIX A: BOUNDARY CONDITION (III)}

The boundary condition can be found with the help of Fig. 9, which is a zoom in of Fig. 1(b) near the interface (4)/(5)/grain. The positive direction of the locally defined coordinate in each section is marked by arrow. With the help of these arrows, one can write

$$
I_{\sigma}^{(4)}=I_{\sigma}^{G}+I_{\sigma}^{(5)},
$$

i.e., the current flowing into the node equals to those flowing out. The current density is related to electrochemical poten-

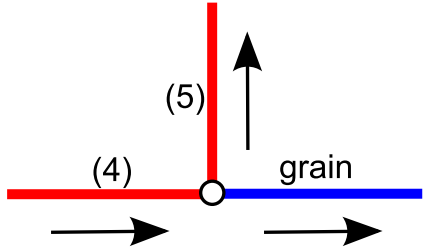

FIG. 9. (Color online) The zoom in of Fig. 1(b) near the interface $(4) /(5) /$ grain. Arrows mark the locally defined positive direction in each region. Three regions meet at the central node. In the local coordinates of (4), the node is at $L$. In the local coordinates of (5), the node is at 0 .

tial by $j_{\sigma}=-\left(\sigma_{\sigma} / e\right) \partial_{x} \mu_{\sigma}$. We will take the spin-up component as a example. In (4) and (5), $\sigma_{\uparrow}=\frac{\sigma_{N}}{2}$; and the spin-up current flowing from the node to the grain is defined as $I_{\uparrow}^{G} \equiv I^{G}$. Put these together, one can easily obtain that

$$
-A_{\square} \frac{\sigma_{N}}{2 e} \partial_{x} \mu_{\uparrow}^{(4)}(L)=I^{G}-A_{\square} \frac{\sigma_{N}}{2 e} \partial_{x} \mu_{\uparrow}^{(5)}(0) .
$$

Similarly, for spin-down $I_{\downarrow}^{G} \equiv-I^{G}$, so

$$
-A_{\square} \frac{\sigma_{N}}{2 e} \partial_{x} \mu_{\downarrow}^{(4)}(L)=-I^{G}-A_{\square} \frac{\sigma_{N}}{2 e} \partial_{x} \mu_{\downarrow}^{(5)}(0)
$$

\section{APPENDIX B: THE QUANTUM THEORY OF THE FERROMAGNETIC NANOGRAIN}

\section{Model of ferromagnetic nanograin}

In this work, we will describe the ferromagnetic nanograin by using the minimal possible mode ${ }^{45,46}$ proposed to describe the experiment transport spectra through cobalt nanograin..$^{8,9}$ This model was then adopted to discuss spinpolarized current-induced relaxation and spin torque. ${ }^{16,17,44} \mathrm{It}$ also provided a starting point to study the Kondo resonance in the scanning tunnel microscope spectrum of a ferromagnetic cluster on metal surface ${ }^{56} \mathrm{~A}$ more detailed microscopic tight-binding model with exchange interactions and atomic spin-orbit couplings was also proposed, ${ }^{57-59}$ to reveal a unified origin of the magnetic anisotropy as well as collective and quasiparticle excitations in the ferromagnetic nanograin. Besides, the Jaynes-Cummings model also reproduced the transport features by considering the interaction between particle-hole excitation and magnon. ${ }^{60}$

In this work, we focus on how the collective spin and one-particle excitations of the grain react to the spin bias, therefore the minimal model is adequate for the current topic. The Hamiltonian for the grain and its couplings to nearby leads is given by

$$
H_{\text {total }}=H_{G}+H_{\text {lead }}+H_{\mathrm{T}} \text {, }
$$

where the Hamiltonian for the ferromagnetic grain takes the form, 


$$
H_{G}=\sum_{i \sigma} \epsilon_{i \sigma} d_{i \sigma}^{\dagger} d_{i \sigma}-\frac{J}{2} \hat{\mathbf{S}} \cdot \hat{\mathbf{S}}-\frac{K_{N}}{S_{0}} \hat{S}_{z}^{2}+E_{C} \delta \hat{N}^{2}+V_{g} \delta \hat{N}
$$

where the first term stands for the kinetic energy of electrons in the grain, $d_{i \sigma}\left(d_{i \sigma}^{\dagger}\right)$ annihilates (creates) an electron on the one-particle level $i$ in the grain, with energy $\epsilon_{i \sigma}$ and spin $\sigma$ $=\{\uparrow, \downarrow\} . \hat{\mathbf{S}}=\sum_{i} \frac{1}{2} \sum_{\sigma \sigma^{\prime}} d_{i \sigma^{\dagger}}^{\dagger} \vec{\tau}_{\sigma \sigma^{\prime}} d_{i \sigma^{\prime}}$ is the total angular momentum of the grain electrons, $\vec{\tau}$ is the vector of Pauli matrices. $J$ is a phenomenological constant depicting the exchange interactions between each pair of electrons in the grain. $\hat{S}_{z}$ is the $z$ component of $\hat{\mathbf{S}}$. $K_{N}$ is the volume-independent anisotropic constant. In this work, we consider that the fluctuation of the electron number $(\sim 1)$ is much smaller than the itinerant electrons $>1000$ already in the grain, so the fluctuation of $K_{N}$ as a function of the electron number is ignored. $\delta N$ is the number of extra electrons added into the grain, compared with a reference electron number $N_{0}$ already in the grain. $E_{C}$ is the charging energy required to add the excess electrons in the grain, which we will see later can be compensated by applying a gate voltage $V_{g}$.

We refer the part where regions (4), (5), and (6) connect the grain as two "leads," one is from regions (4) and (5) together, and the other from region (6). For convenience, we call them lead $(4,5)$ and lead (6), respectively. The Hamiltonian for the leads takes the form

$$
H_{\text {lead }}=\sum_{k, \alpha, \tau} \epsilon_{k \alpha} c_{k \alpha \tau}^{\dagger} c_{k \alpha \tau}
$$

where $c_{k \alpha \tau}^{\dagger}\left(c_{k \alpha \tau}\right)$ is the creation (annihilation) operator for a continuous state in the lead $\alpha \in\{(4,5),(6)\}$ with energy $\epsilon_{k \alpha}$ and spin $\tau \in\{+,-\}$. The tunneling between the grain and the leads is described by

$$
\begin{aligned}
H_{\mathrm{T}}= & \sum_{k, \alpha, i} V_{k \alpha}\left[\left(\cos \frac{\theta_{\alpha}}{2} c_{k \alpha+}^{\dagger}-\sin \frac{\theta_{\alpha}}{2} c_{k \alpha-}^{\dagger}\right) d_{i \uparrow}\right. \\
& \left.+\left(\sin \frac{\theta_{\alpha}}{2} c_{k \alpha+}^{\dagger}+\cos \frac{\theta_{\alpha}}{2} c_{k \alpha-}^{\dagger}\right) d_{i \downarrow}\right]+ \text { H.c. },
\end{aligned}
$$

where $\theta_{\alpha}$ is the angle between the easy axis of the grain and the spin-quantization direction of the lead $\alpha$. For simplicity, we set the easy axis of the grain as $z$ axis and assume that $\theta_{(6)}=0$ and $\theta_{(4,5)}=\theta \in[0, \pi / 2]$, as shown in Fig. 4. In the following, we denote $\mu_{+/-}^{(4)}=\mu_{\uparrow / \downarrow}^{(4)}$ when $\theta_{(4,5)}=0$ for simplicity.

\section{Ground branch $\left|N_{0}, S_{0}, S_{z}\right\rangle$ of the grain and possible excitations}

The eigenstates of $H_{G}$ are labeled by $\left|\left\{n_{i}\right\}, S, S_{z}\right\rangle$, where $\left\{n_{i}\right\}$ denote the occupation on each level in the grain, $S$ and $S_{z}$ are, respectively, the quantum numbers for the magnitude of $\hat{\mathbf{S}}$ and $\hat{S}_{z}$. Because of the low experimental temperature (as low as $20 \mathrm{mK})$ and the large Coulomb repulsion $E_{C}($ $>30 \mathrm{meV}),{ }^{8}$ the charge fluctuation and particle-hole excitations [Fig. 10(c)] are suppressed hence it is reasonable to assume that the electrons in the grain compactly occupy all the lowest available levels. These states are denoted as
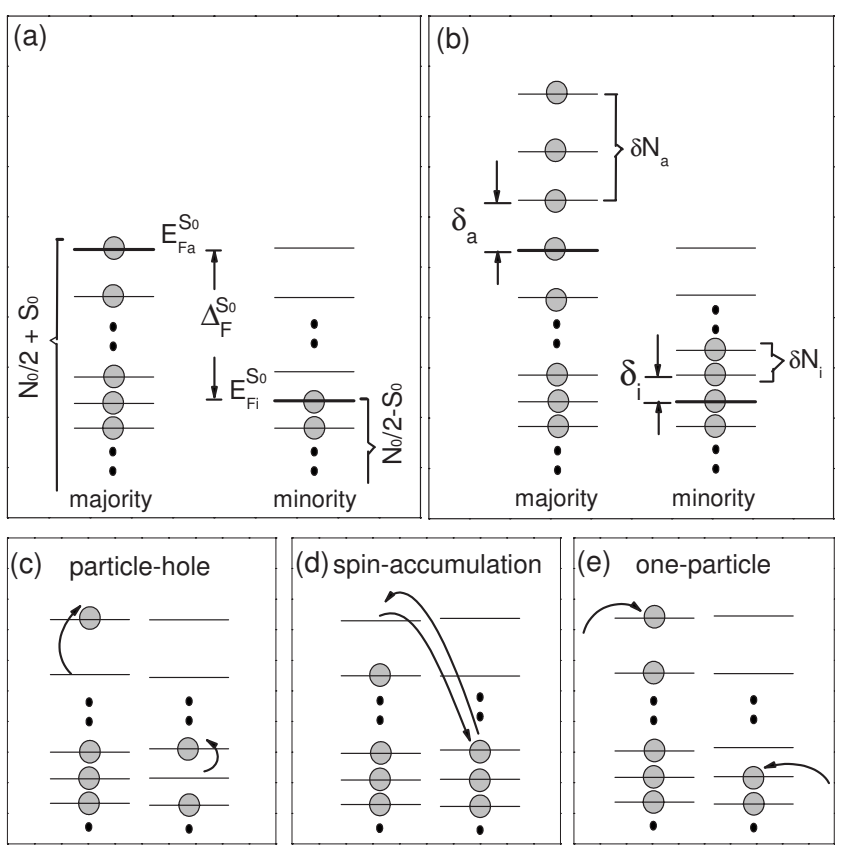

FIG. 10. The filling of one-particle levels in the majority and minority bands of the grain with (a) $N_{0}$ electrons and (b) $N_{0}+\delta N$ electrons, where $\delta N=\delta N_{a}+\delta N_{i}$, and $\delta N_{a}$ and $\delta N_{i}$ are the excess electrons added to the majority and minority band, respectively. For the ground branch with $N_{0}$ electrons in the grain, the magnitude of the angular momentum $S=S_{0} . E_{F a}^{S_{0}}$, and $E_{F i}^{S_{0}}$ are the highest occupied levels of the majority and minority bands for the ground branch for $N=N_{0}$ and $S=S_{0} . \delta_{a}$ and $\delta_{i}$ is the level spacings near the $E_{F a}^{S_{0}}$ and $E_{F i}^{S_{0}}$, respectively. (c) The particle-hole excitations, with excitation energy $\sim \delta_{i}, \delta_{a}>1 \mathrm{meV}$. (d) The spin-accumulation excitations, with excitation energy $\sim J \sim 2 \mathrm{meV}$. These two excitations are high-energy excitations, with the excitation energy larger than the spin bias $V_{s}$, thus cannot be excited and will be omitted. Note that according to Fig. 3, $V_{s} \sim 0.1 \mathrm{meV}$. (e) One-particle excitations that changes the electron number by one. It can add extra electrons to the majority or minority band. By tuning the gate voltage, its excitation energy can be minimized down to $\sim K_{N} \sim 0.01 \mathrm{meV}$, which is smaller than the spin bias $V_{s} \sim 0.1 \mathrm{meV}$. Then, one-particle excitations is the only possible excitation for the present device and parameters.

$\left|N, S, S_{z}\right\rangle$, where $N=\sum_{i} n_{i}$ is the total electron number in the grain.

When there is $N=N_{0}$ electrons in the grain, the competition between the kinetic energy and the $J$ term will force $N_{0} / 2+S_{0}$ electrons (the majority band) to orient antiparallel with the rest $N_{0} / 2-S_{0}$ electrons (the minority band), leading to a nonzero magnitude of angular momentum $S=S_{0}$ at the ground states (Stoner instability), as shown in Figs. 4 and 10(a). This overall ground branch is denoted as $\left|N_{0}, S_{0}, S_{z}\right\rangle$, where $S_{z} \in\left[-S_{0}, S_{0}\right]$. The $\left(2 S_{0}+1\right)$-fold degeneracy of the overall ground branch is lifted by the anisotropy, with two degenerate ground states $\left|N_{0}, S_{0}, \pm S_{0}\right\rangle$.

There are three kinds of basic excitations from the ground branches $\left|N_{0}, S_{0}, S_{z}\right\rangle$, as shown in Figs. 10(c)-10(e). The particle-hole excitation destroys the compact occupation of the one-particle levels, while does not change $N, S$, and $S_{z}$. The spin-accumulation excitation changes $S$ by moving electron between the majority and minority bands, while does 
not change $N_{0}$ and $S_{z}$. The one-particle excitation changes $N$ by adding or removing electrons, which also leads to changes in $S$ and $S_{z}$.

Generally, the excited energy from $\left|N_{0}, S_{0}, S_{z}\right\rangle$ to $\mid N_{0}$ $\left.+\delta N, S_{0}+\delta S, S_{z}+\delta S_{z}\right\rangle$ can be found with the help of Figs. 10 (a) and 10(b) as [using the relations $\delta N=\delta N_{a}+\delta N_{i}$ and $\left.\delta S=\left(\delta N_{a}-\delta N_{i}\right) / 2\right],{ }^{45,61}$

$$
\begin{aligned}
\delta E\left(\delta N, \delta S, \delta S_{z}\right) & \\
\equiv & E_{\left|N_{0}+\delta N, S_{0}+\delta S, S_{z}+\delta S_{z}\right\rangle}-E_{\left|N_{0}, S_{0}, S_{z}\right\rangle} \\
= & (\delta N)^{2}\left[E_{C}+\frac{1}{8} \delta_{a}+\frac{1}{8} \delta_{i}\right]+\delta N\left[\bar{E}_{F}^{S_{0}}+\frac{1}{4} \delta_{a}+\frac{1}{4} \delta_{i}+V_{g}\right] \\
& +(\delta S)^{2}\left[\frac{\delta_{a}}{2}+\frac{\delta_{i}}{2}-\frac{J}{2}\right] \\
& +\delta S\left[\frac{\delta_{a}}{2}-\frac{\delta_{i}}{2}+\Delta_{F}^{S_{0}}-J\left(S_{0}+\frac{1}{2}\right)\right] \\
& +\delta N \delta S\left[\frac{1}{2} \delta_{a}-\frac{1}{2} \delta_{i}\right]-\frac{K_{N}}{S_{0}}\left(2 S_{z} \delta S_{z}+\delta S_{z}^{2}\right)
\end{aligned}
$$

where the definitions of parameters are given in Fig. 10 and $\bar{E}_{F}^{S_{0}}=\left(E_{F a}^{S_{0}}+E_{F i}^{S_{0}}\right) / 2$.

We will adopt a set of parameters for a grain with $S_{0}$ $=1000$, as given in Table. I. The theoretical calculations ${ }^{45,46,61}$ based on these parameters are consistent with most features observed in the experimental transport spectra. ${ }^{8,9}$ With these parameters, the value of $J$ can be deduced using a saddle-point analysis as follows.

\section{Range of $\boldsymbol{J}$ for a stable $S_{\mathbf{0}}$ by saddle-point analysis}

Above we just assume that $S=S_{0}$ when there are $N=N_{0}$ electrons in the grain. Since $S_{0}$ originates from the competition between the $J$ term and the kinetic energy, its value should be calculated for the given values of $J$ and $\delta_{a, i}$. However, since we already know $S_{0} \sim 1000$ from the experiments, ${ }^{8-11}$ and $\delta_{a, i}$ from the band calculation, ${ }^{62}$ we will use these data to conversely deduce the value of $J$, then generate other information (e.g., one-particle excitations) at the proximity of the deduced $J$, much like a saddle-point analysis.

The stability of the branch $\left|N=N_{0}, S=S_{0}\right\rangle$ requires that its energy should be at least locally minimal compared to the states $\left|N=N_{0}, S=S_{0} \pm 1\right\rangle$, i.e.,

$$
\begin{gathered}
\delta E(\delta N=0, \delta S=+1)=\delta_{a}+\Delta_{F}^{S_{0}}-J\left(S_{0}+1\right) \geq 0, \\
\delta E(\delta N=0, \delta S=-1)=\delta_{i}-\Delta_{F}^{S_{0}}+J S_{0} \geq 0,
\end{gathered}
$$

where we have used Eq. (B5) and ignored the anisotropy term because $K_{N}$ is much smaller than other energies. In other words, Eq. (B6) leads to that, for $J$ belongs to the range

$$
\frac{\Delta_{F}^{S_{0}}-\delta_{i}}{S_{0}} \leq J \leq \frac{\Delta_{F}^{S_{0}}+\delta_{a}}{S_{0}+1},
$$

the grain will adopt a stable $S=S_{0}$ when there are $N_{0}$ electrons in the grain. Once $J$ exceeds this range, the overall ground branch will evolve to adopt a smaller or larger $S$ $=S_{0}^{\prime}=S_{0} \mp 1$, then Eq. (B7) still holds for the new $S_{0}^{\prime}$ (note

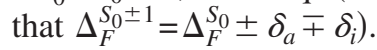

\section{One-particle excitations to the branches $\left|N_{0}+1, S_{0} \pm 1 / 2, S_{z}\right\rangle$}

The excitation energies for the particle-hole excitation shown in Fig. 10(c) and spin-accumulation excitation shown in Fig. 10(d) are of the order of $\delta_{a / i}$ and $J$, respectively. ${ }^{17,45,61}$ According to the parameters for $S_{0}=1000$ shown in Table I, these excitations are of $\mathrm{meV}$, much larger than the $V_{s}$ $\sim 0.05$ meV estimated in Fig. 3, thus will be excluded. In the following, we will only consider the one-particle excitation from the $N_{0}$ to $N_{0}+1$ electrons, as shown by Fig. 10(e), i.e., adding excess electrons to the grain.

Again, because $E_{C} \gg k_{B} T$, the gate voltage in this work is restricted so that one and only one excess electron $(\delta N=1)$ can be added into the grain. Depending on this excess electron being added to the majority or minority band, the magnitude of the angular momentum could change to $S=S_{0}$ $+1 / 2$ or $S_{0}-1 / 2$, respectively. With the help of Eqs. (B5) and (B7), we find that, when

$$
\frac{\Delta_{F}^{S_{0}}-\delta_{i}}{S_{0}} \leq J \leq \frac{\Delta_{F}^{S_{0}}+\delta_{a}-\delta_{i}}{S_{0}+1 / 2},
$$

the $N_{0}+1$-electron ground branch will adopt $S=S_{0}-1 / 2$, and the required transition energies from $\left|N_{0}, S_{0}, S_{z}\right\rangle$ are

$$
\begin{aligned}
& E_{\left|N_{0}+1, S_{0}-1 / 2, S_{z} \pm 1 / 2\right\rangle}-E_{\left|N_{0}, S_{0}, S_{z}\right\rangle} \\
& \quad=\delta_{i}+V_{g}+E_{C}+E_{F i}^{S_{0}}+\frac{J}{2}\left(S_{0}+\frac{1}{4}\right)-\frac{K_{N}}{S_{0}}\left(\frac{1}{4} \pm S_{z}\right) .
\end{aligned}
$$

On the contrary, when

$$
\frac{\Delta_{F}^{S_{0}}+\delta_{a}-\delta_{i}}{S_{0}+1 / 2} \leq J \leq \frac{\Delta_{F}^{S_{0}}+\delta_{a}}{S_{0}+1},
$$

the $N_{0}+1$-electron ground branch will adopt $S=S_{0}+1 / 2$, and the required transition energies from $\left|N_{0}, S_{0}, S_{z}\right\rangle$ are

$$
\begin{aligned}
& E_{\left|N_{0}+1, S_{0}+1 / 2, S_{z} \pm 1 / 2\right\rangle}-E_{\left|N_{0}, S_{0}, S_{z}\right\rangle} \\
& \quad=\delta_{a}+V_{g}+E_{C}+E_{F a}^{S_{0}}-\frac{J}{2}\left(S_{0}+\frac{3}{4}\right)-\frac{K_{N}}{S_{0}}\left(\frac{1}{4} \pm S_{z}\right) .
\end{aligned}
$$

To summarize the above saddle-point analysis, the angular momenta of $N_{0^{-}}$and $\left(N_{0}+1\right)$-electron ground branches as a function of $J$ are shown in Fig. 11(a), in which the arrows mark the ranges indicated by Eqs. (B7), (B8), and (B10).

According to Fig. 11(a), for most value of $J, S$ of the $\left(N_{0}+1\right)$-electron branch is $1 / 2$ smaller than that of the $N_{0}$-electron branch. This is a direct results of $\delta_{i}<\delta_{a}$. Although $J$ is not a tunable quantity, Fig. 11(a) implies that in reality the excess electron is far more likely to occupy the minority band than the majority band, which is also consistent with the previous literatures. ${ }^{16,45}$ Therefore, in the following we will mainly consider the one-particle excitations 


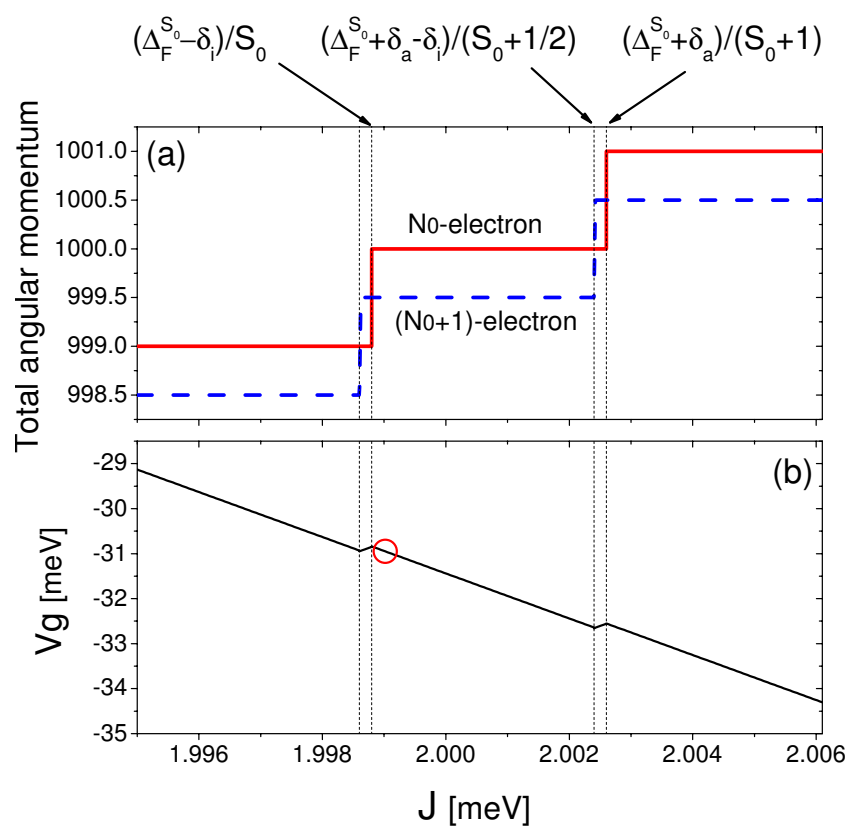

FIG. 11. (Color online) (a) Total angular momenta for the ground branches of the grain with $N_{0}$ and $N_{0}+1$ electrons, respectively. (b) Along the zigzag line of $\left(J, V_{g}\right)$, the $N_{0^{-}}$and $\left(N_{0}+1\right)$-electron ground branches are nearly degenerate. The parameters are listed in Table I.

between the ground branches $\left|N_{0}, S_{0}, S_{z}\right\rangle$ and $\mid N_{0}+1, S_{0}$ $\left.-1 / 2, S_{z}\right\rangle$.

\section{States used for numerical simulations}

Remember we have set $\mu^{(5)}(\infty)$ as the energy zero point. With respect to $\mu^{(5)}(\infty)$, we can always choose suitable $V_{g}$ in Eqs. (B9) and (B11) to compensate $E_{C}$ and other energies, so that the ground branches with $N_{0}$ and $N_{0}+1$ electrons can be tuned to be nearly degenerate. These $V_{g}$ as a function of $J$ are shown in Fig. 11(b). For instance, in Eq. (B9), by choosing $V_{g}=-\delta_{i}-E_{C}-E_{F i}^{S_{0}}-J\left(S_{0}+1 / 4\right) / 2+K_{N} / 4 S_{0}$, one obtains

$$
E_{\left|N_{0}+1, S_{0}-1 / 2, S_{z} \pm 1 / 2\right\rangle}-E_{\left|N_{0}, S_{0}, S_{z}\right\rangle}=\mp \frac{K_{N}}{S_{0}} S_{z} .
$$

In this context, the energy required to add an electron from lead $(4,5)$ into the grain is related to only the magnetization of the grain. According to Eq. (B12), the spectrum width of $E_{\left|N_{0}+1, S_{0}-1 / 2, S_{z} \pm 1 / 2\right\rangle}-E_{\left|N_{0}, S_{0}, S_{z}\right\rangle}$ is $2 K_{N}$ for all the possible $S_{z}$ $\in\left[-S_{0}, S_{0}\right]$. This value is the key to determine the critical $I_{c}$ in the Sec. IV A.

Experimentally, it is easy to find the suitable $V_{g}$ at which the ground branches with $N_{0}$ and $N_{0}+1$ electrons are nearly degenerate, as in the usual transport experiments. ${ }^{8,9}$ For example, one just apply a small charge bias voltage $>2 K_{N}$ between lead (4,5) and lead (6), and measure the current through the grain while scanning $V_{g}$, like a usual sourcegate-drain measurement. Because of the Coulomb blockade, the grain cannot conduct electrons unless the $N_{0^{-}}$and $\left(N_{0}\right.$ $+1)$-electron ground branches are degenerate. Therefore, the nearly degenerate situation is find as: at which $V_{g}$, the grain is conducting under a small charge bias voltage between lead $(4,5)$ and lead $(6)$.

Based on the above analysis and discussions from Appendixes B 2-B 5, in the following, we will consider mainly the one-particle excitations from the branches $\left|N_{0}, S_{0}, S_{z}\right\rangle$ to $\mid N_{0}$ $\left.+1, S_{0}-1 / 2, S_{z}\right\rangle$ and when their energies are nearly degenerate. Specifically, we will choose a set of $\left(J, V_{g}\right)$ marked by the circle in Fig. 11(b) for our numerical simulations, where $J=\Delta_{F}^{S_{0}} /\left(S_{0}+1 / 2\right), \quad$ and $\quad V_{g}=-\delta_{i}-E_{C}-E_{F i}^{S_{0}}-J\left(S_{0}+1 / 4\right) / 2$ $+K_{N} / 4 S_{0}+\Delta V_{g}, \Delta V_{g}$ is a small variation in the gate voltage that drives the grain away from the nearly degenerate point of $N_{0^{-}}$and $\left(N_{0}+1\right)$-electron ground branches.

\section{APPENDIX C: VALIDITY OF RATE EQUATIONS}

Although the rate equations formalism is widely employed for the mesoscopic systems weakly coupled to the electrodes, its validity deserves some discussion. ${ }^{52,54}$ In the previous works by Waintal et al., ${ }^{16,17}$ the intrinsic spin relaxation is considered in terms of coupling to a bosonic bath. We do not consider this effect for two reasons: (i) according to Eq. (3) of Ref. 16, the intrinsic relaxation will lead to a term similar to the Gilbert damping, which tends to relax the grain to one of its two degenerate maximally magnetized ground states, e.g., $\left|N_{0}, S_{0},-S_{0}\right\rangle$ or $\left|N_{0}, S_{0}, S_{0}\right\rangle$. In the following, we will mainly discuss how to use the NLSV signal to read out the magnetization of the grain (Sec. III), which already limits the discussion to these maximally magnetized states. Therefore, the results in Sec. III will be qualitatively unaffected by the intrinsic relaxation. (ii) For the spin biasinduced magnetization switching discussed in Sec. IV, the results are only valid when the coupling between the grain and the lead is dominant and much smaller in time scale than the intrinsic relaxation. On the other hand, the Born and Markoff approximations enforce that $\Gamma$ is much smaller than $k_{B} T$ (bath temperature) and the energy difference between many-body states in the grain. These two requirements confine the validity range of $\Gamma$ in this work.

Besides, the particle-hole excitation terms in Eq. (10) of Ref. 61 are also absent in this work, because the energy scale of these excitations are of the order $\delta_{a}, \delta_{i}>1 \mathrm{meV}$, which already one order larger than the spin bias $\left(V_{s} \sim 0.1 \mathrm{meV}\right)$ that can be generated according to Fig. 3 . 
${ }^{1}$ J. C. Slonczewski, J. Magn. Magn. Mater. 159, L1 (1996).

${ }^{2}$ L. Berger, Phys. Rev. B 54, 9353 (1996).

${ }^{3}$ M. Tsoi, A. G. M. Jansen, J. Bass, W. C. Chiang, M. Seck, V. Tsoi, and P. Wyder, Phys. Rev. Lett. 80, 4281 (1998).

${ }^{4}$ E. B. Myers, D. C. Ralph, J. A. Katine, R. N. Louie, and R. A. Buhrman, Science 285, 867 (1999).

${ }^{5}$ J. Z. Sun, J. Magn. Magn. Mater. 202, 157 (1999).

${ }^{6}$ J. A. Katine, F. J. Albert, R. A. Buhrman, E. B. Myers, and D. C. Ralph, Phys. Rev. Lett. 84, 3149 (2000).

${ }^{7}$ Y. Jiang, S. Abe, T. Ochiai, T. Nozaki, A. Hirohata, N. Tezuka, and K. Inomata, Phys. Rev. Lett. 92, 167204 (2004).

${ }^{8}$ S. Guéron, M. M. Deshmukh, E. B. Myers, and D. C. Ralph, Phys. Rev. Lett. 83, 4148 (1999).

${ }^{9}$ M. M. Deshmukh, S. Kleff, S. Guéron, E. Bonet, A. N. Pasupathy, J. von Delft, and D. C. Ralph, Phys. Rev. Lett. 87, 226801 (2001).

${ }^{10}$ M. Jamet, W. Wernsdorfer, C. Thirion, D. Mailly, V. Dupuis, P. Mélinon, and A. Pérez, Phys. Rev. Lett. 86, 4676 (2001).

${ }^{11}$ C. Thirion, W. Wernsdorfer, and D. Mailly, Nature Mater. 2, 524 (2003).

${ }^{12}$ J. I. Inoue and A. Brataas, Phys. Rev. B 70, 140406(R) (2004).

${ }^{13}$ M. Braun, J. König, and J. Martinek, Phys. Rev. B 70, 195345 (2004).

${ }^{14}$ W. Wetzels, G. E. W. Bauer, and M. Grifoni, Phys. Rev. B 72, 020407(R) (2005).

${ }^{15}$ M. B. A. Jalil and S. G. Tan, Phys. Rev. B 72, 214417 (2005).

${ }^{16}$ X. Waintal and O. Parcollet, Phys. Rev. Lett. 94, 247206 (2005).

${ }^{17}$ O. Parcollet and X. Waintal, Phys. Rev. B 73, 144420 (2006).

${ }^{18}$ J. Fernández-Rossier and R. Aguado, Phys. Rev. Lett. 98, 106805 (2007).

${ }^{19}$ X. R. Wang and Z. Z. Sun, Phys. Rev. Lett. 98, 077201 (2007).

${ }^{20}$ H.-Z. Lu and S.-Q. Shen, Phys. Rev. B 77, 235309 (2008).

${ }^{21}$ H.-Z. Lu, B. Zhou, and S.-Q. Shen, Phys. Rev. B 79, 174419 (2009).

${ }^{22}$ T. Y. Chen, S. X. Huang, C. L. Chien, and M. D. Stiles, Phys. Rev. Lett. 96, 207203 (2006).

${ }^{23}$ S. Krause, L. Berbil-Bautista, G. Herzog, M. Bode, and R. Wiesendanger, Science 317, 1537 (2007).

${ }^{24}$ X. J. Wang, H. Zou, and Y. Ji, Appl. Phys. Lett. 93, 162501 (2008).

${ }^{25}$ S. Garzon, L. Ye, R. A. Webb, T. M. Crawford, M. Covington, and S. Kaka, Phys. Rev. B 78, 180401(R) (2008).

${ }^{26}$ D. Ralph and M. D. Stiles, J. Magn. Magn. Mater. 320, 1190 (2008).

${ }^{27}$ M. Johnson and R. H. Silsbee, Phys. Rev. Lett. 55, 1790 (1985).

${ }^{28}$ A. T. Filip, B. H. Hoving, F. J. Jedema, B. J. van Wees, B. Dutta, and S. Borghs, Phys. Rev. B 62, 9996 (2000).

${ }^{29}$ F. J. Jedema, A. T. Filip, and B. J. van Wees, Nature (London) 410, 345 (2001).

${ }^{30}$ S. O. Valenzuela and M. Tinkham, Appl. Phys. Lett. 85, 5914 (2004).

${ }^{31}$ Y. Ji, A. Hoffmann, J. S. Jiang, and S. D. Bader, Appl. Phys. Lett. 85, 6218 (2004).

${ }^{32}$ D. Beckmann, H. B. Weber, and H. v. Löhneysen, Phys. Rev.
Lett. 93, 197003 (2004).

${ }^{33}$ T. Kimura, J. Hamrle, Y. Otani, K. Tsukagoshi, and Y. Aoyagi, Appl. Phys. Lett. 85, 3501 (2004).

${ }^{34}$ S. Garzon, I. Zutic, and R. A. Webb, Phys. Rev. Lett. 94, 176601 (2005).

${ }^{35}$ A. Brataas, G. E. Bauer, and P. J. Kelly, Phys. Rep. 427, 157 (2006).

${ }^{36}$ T. Kimura, Y. Otani, and J. Hamrle, Phys. Rev. Lett. 96, 037201 (2006).

${ }^{37}$ T. Yang, T. Kimura, and Y. Otani, Nat. Phys. 4, 851 (2008).

${ }^{38}$ P. C. van Son, H. van Kempen, and P. Wyder, Phys. Rev. Lett. 58, 2271 (1987).

${ }^{39}$ T. Valet and A. Fert, Phys. Rev. B 48, 7099 (1993).

${ }^{40}$ S. Hershfield and H. L. Zhao, Phys. Rev. B 56, 3296 (1997).

${ }^{41}$ F. J. Jedema, M. S. Nijboer, A. T. Filip, and B. J. van Wees, Phys. Rev. B 67, 085319 (2003).

${ }^{42}$ S. Takahashi and S. Maekawa, Phys. Rev. B 67, 052409 (2003).

${ }^{43}$ J. Z. Sun, Phys. Rev. B 62, 570 (2000).

${ }^{44}$ X. Waintal and P. W. Brouwer, Phys. Rev. Lett. 91, 247201 (2003).

${ }^{45}$ C. M. Canali and A. H. MacDonald, Phys. Rev. Lett. 85, 5623 (2000).

${ }^{46}$ S. Kleff, J. von Delft, M. M. Deshmukh, and D. C. Ralph, Phys. Rev. B 64, 220401(R) (2001).

${ }^{47}$ D.-K. Wang, Q.-F. Sun, and H. Guo, Phys. Rev. B 69, 205312 (2004).

${ }^{48}$ Q.-F. Sun, Y. Xing, and S. Q. Shen, Phys. Rev. B 77, 195313 (2008).

${ }^{49}$ Y. Xing, Q.-F. Sun, and J. Wang, Appl. Phys. Lett. 93, 142107 (2008).

${ }^{50}$ G. Stefanucci, E. Perfetto, and M. Cini, Phys. Rev. B 78, 075425 (2008).

${ }^{51}$ J. Bass and W. P. P., Jr., J. Phys.: Condens. Matter 19, 183201 (2007).

${ }^{52}$ C. W. J. Beenakker, Phys. Rev. B 44, 1646 (1991).

${ }^{53}$ K. Blum, Density Matrix Theory and Applications (Plenum, New York, 1996).

${ }^{54}$ J. von Delft and D. C. Ralph, Phys. Rep. 345, 61 (2001).

${ }^{55}$ T. Kimura, Y. C. Otani, and P. M. Levy, Phys. Rev. Lett. 99, 166601 (2007).

${ }^{56}$ G. A. Fiete, G. Zarand, B. I. Halperin, and Y. Oreg, Phys. Rev. B 66, 024431 (2002).

${ }^{57}$ A. H. MacDonald and C. M. Canali, Solid State Commun. 119, 253 (2001).

${ }^{58}$ A. Cehovin, C. M. Canali, and A. H. MacDonald, Phys. Rev. B 66, 094430 (2002).

${ }^{59}$ A. Cehovin, C. M. Canali, and A. H. MacDonald, Phys. Rev. B 68, 014423 (2003).

${ }^{60}$ L. Michalak, C. M. Canali, and V. G. Benza, Phys. Rev. Lett. 97, 096804 (2006)

${ }^{61}$ S. Kleff and J. von Delft, Phys. Rev. B 65, 214421 (2002).

${ }^{62}$ D. A. Papaconstantopoulos, Handbook of the Band Structure of Elemental Solids (Plenum, New York, 1986). 